This item was submitted to Loughborough's Research Repository by the author.

Items in Figshare are protected by copyright, with all rights reserved, unless otherwise indicated.

\title{
New generalized almost perfect nonlinear functions
}

PLEASE CITE THE PUBLISHED VERSION

https://doi.org/10.1016/j.ffa.2020.101796

PUBLISHER

Elsevier

VERSION

AM (Accepted Manuscript)

PUBLISHER STATEMENT

This paper was accepted for publication in the journal Finite Fields and Their Applications and the definitive published version is available at https://doi.org/10.1016/j.ffa.2020.101796.

\section{LICENCE}

CC BY-NC-ND 4.0

\section{REPOSITORY RECORD}

Özbudak, Ferruh, and Ana Salagean. 2020. "New Generalized Almost Perfect Nonlinear Functions". Loughborough University. https://hdl.handle.net/2134/13593596.v1. 


\title{
New Generalized Almost Perfect Nonlinear Functions
}

\author{
Ferruh Özbudak ${ }^{1}$ and Ana Sălăgean ${ }^{2}$ \\ 1 Department of Mathematics and Institute of Applied Mathematics, Middle East \\ Technical University, Ankara, Turkey e-mail: ozbudak@metu.edu.tr \\ 2 Department of Computer Science, Loughborough University, Loughborough, UK \\ e-mail: a.m.salagean@lboro.ac.uk
}

\begin{abstract}
APN (almost perfect non-linear) functions over finite fields of even characteristic are widely studied due to their applications to the design of symmetric ciphers resistant to differential attacks. This notion was recently generalized to GAPN (generalized APN) functions by Kuroda and Tsujie to odd characteristic $p$. They presented some constructions of GAPN functions, and other constructions were given by Zha et al. We present new constructions of GAPN functions both in the case of monomial and multinomial functions. Our monomial GAPN functions can be viewed as a further generalization of the Gold APN functions. We show that a certain technique used by Hou to construct permutations over finite fields also yields monomial GAPN functions. We also present several new constructions of GAPN functions which are sums of monomial GAPN functions, as well as new GAPN functions of degree $p$ which can be written as the product of two powers of linearized polynomials. For this latter construction we describe some interesting differences between even and odd characteristic and also obtain a classification in certain cases.
\end{abstract}

Keywords: APN function; GAPN function; Discrete derivative; Linearized polynomial (MSC codes: 94A60, 11T71, 11T06)

\section{Introduction}

Differential cryptanalysis was introduced by Biham and Shamir in [2] in order to break symmetric ciphers using the advantage of non-uniform distribution of ciphertext differences corresponding to plaintext differences. This publication stimulated a lot of research on the study of security offered by different types of functions regarding differential attacks. These functions are Boolean functions over a finite field of order $2^{n}$ and they correspond to the Substitution box (S-box) of a block cipher. The security of a block cipher against a differential attack is measured by the differential uniformity of the Boolean function of its S-box. A Boolean function having the best (i.e. smallest) differential uniformity is called almost perfect nonlinear (APN) function, which was introduced by Nyberg [12]. There are many applications and connections of APN functions to areas including 
design theory, finite geometry and cryptography. The research on APN functions is still developing rapidly and we refer to the interesting survey papers $[3,13]$ and the references therein. We note that higher order discrete derivatives are also used for attacking symmetric ciphers, for example the AIDA and the cube attack $[4,15]$ and their generalization to characteristic $p$ [14].

Recently Kuroda and Tsujie presented a new generalization of APN functions to odd characteristic [9]. They introduced the notion of generalized almost perfect function (GAPN) for arbitrary finite fields, which corresponds to APN functions in even characteristic. They gave interesting examples and some applications including cryptography and dual arcs of finite geometry. Kuroda [8] and Zha et al. [16] continued this work and they not only presented new classes of GAPN functions but also classified some of GAPN classes.

In this paper we obtain further results on GAPN functions. In Section 2 we give some preliminaries including a reinterpretation of the GAPN notion using higher discrete derivatives and our definition of generalized extended affine equivalence, which is more natural to use than extended affine equivalence (EAequivalence) in the context of GAPN functions. We also present a new characterization of GAPN monomials of algebraic degree $p$, which is easier to check algorithmically then existing ones (see Theorem 1).

Section 3 is devoted to the construction of a large class of new GAPN monomials (see Theorem 2) which can be considered a further generalisation of the Gold functions. Namely, the original APN Gold functions are functions over $\mathbb{F}_{2^{n}}$ of the form $f(x)=x^{d}$ with $d=1+p^{\ell}$ and $\operatorname{gcd}(\ell, n)=1$. The GAPN generalized Gold functions described by [9, Theorem 1.2] are functions over $\mathbb{F}_{p^{n}}$ of the form $f(x)=x^{d}$ with $d=(p-1)+p^{\ell}$ and $\operatorname{gcd}(\ell, n)=1$. We further generalize them to exponents of the form $d=k_{1}+k_{2} p^{\ell}$, with $\operatorname{gcd}\left(k_{1}+k_{2}-(p-1), p^{n}-1\right)=1$ and $\operatorname{gcd}(\ell, n)=1$. Our construction leads to new examples compared to the existing families in the literature (see $[9,16])$.

In Section 4 we present an interesting connection between GAPN functions and the functions used by Hou [7] as candidates for constructing permutations over $\mathbb{F}_{p^{n}}$, with a focus on the case $p>2$. Essentially, any permutation obtained by their method also produces a monomial GAPN function (see Theorem 3), but not all monomial GAPN functions can be obtained this way (see Theorem 4 and Example 2). The permutations thus obtained in [7] produce GAPN functions which were already known, but further progress in the search of permutations of this type will potentially yield new GAPN functions.

In Section 5 we provide new examples of GAPN functions consisting of a sum of several (up to $p-1$ ) GAPN monomial functions instead of just monomials (see Theorems 5, 6 and 7). The monomials that we add are all generalised Gold functions (in the sense we constructed above) of the same algebraic degree. We also give a full characterisation in the case of a sum of two monomials of this type (Theorem 8). The construction in [16, Proposition 2.7] becomes a particular case of our construction.

The final section concerns some GAPN functions of degree $p$ consisting of many terms. We construct such functions as a product of two functions, one 
being a monomial and the other being a power of a linearized polynomial. For characteristic $p=2$ it is known that, essentially, this technique does not produce any new APN functions compared to the monomial Gold functions (see Berger et al [1]). For odd characteristic Kuroda and Tsujie [9, Proposition 3.5] presented a class of non-monomial GAPN functions constructed in this way, and we present many more. We first give two characterizations of linearized polynomials that produce GAPN functions by the construction above, Theorems 9 and 10, which lead to observing some interesting differences between even and odd characteristics regarding the properties of a class of maps over finite fields studied by Hou [5], see Remark 5. We construct a large class of linearized polynomials (see Theorem 11) which is then used for obtaining a large class of new GAPN functions of algebraic degree $p$ having $(s+1)(s+2) / 2$ distinct terms for all $2 \leq s \leq p-1$ (see Example 3). We also present a necessary and sufficient condition giving, for $n$ odd, a full classification of the GAPN functions of degree $p$ which can be written as the product of a monomial and a power of a binomial of a certain type (see Corollary 3 ).

\section{Preliminaries}

We work over the finite field with $p^{n}$ elements, denoted $\mathbb{F}_{p^{n}}$, with $p$ prime. We recall the definition of generalized almost perfect non-linear functions from [9]:

Definition 1. A function $f: \mathbb{F}_{p^{n}} \rightarrow \mathbb{F}_{p^{n}}$ is called GAPN (generalized almost perfect nonlinear) if for any $a, b \in \mathbb{F}_{p^{n}}, a \neq 0$ the equation

$$
\sum_{i \in \mathbb{F}_{p}} f(x+i a)=b
$$

has at most $p$ solutions.

For $p=2$, this definition becomes the usual definition of APN (almost perfect non-linear) functions over $\mathbb{F}_{2^{n}}$.

We found it convenient to reinterpret the definition of the GAPN using discrete derivatives. The discrete derivative of a function $f: \mathbb{F}_{p^{n}} \rightarrow \mathbb{F}_{p^{n}}$ in direction $a \in \mathbb{F}_{p^{n}}$ is the function $\mathrm{D}_{a} f: \mathbb{F}_{p^{n}} \rightarrow \mathbb{F}_{p^{n}}$ defined as $\mathrm{D}_{a} f(x)=f(x+a)-f(x)$. Higher order derivatives are obtained by repeated differentiation. If we differentiate $k$ times, in directions $a_{1}, \ldots a_{k}$ we will use the notation

$$
\mathrm{D}_{a_{1}, \ldots a_{k}}^{(k)} f=\mathrm{D}_{a_{1}} \mathrm{D}_{a_{2}} \ldots \mathrm{D}_{a_{k}} f .
$$

From the definition of the derivative, one can easily obtain:

$$
\mathrm{D}_{a_{1}, \ldots a_{k}}^{(k)} f=\sum_{i_{1}, \ldots i_{k} \in\{0,1\}}(-1)^{k-i_{1}-\ldots-i_{k}} f\left(x+i_{1} a_{1}+\ldots+i_{k} a_{k}\right)
$$

Note that $a_{1}, \ldots a_{k}$ do not need to be distinct. If $a_{1}=a_{2}=\ldots=a_{k}=a$ then we will write, for short

$$
\mathrm{D}_{a}^{(k)} f=\mathrm{D}_{a, \ldots a}^{(k)} f
$$


and we have

$$
\mathrm{D}_{a}^{(k)} f=\sum_{i=0}^{k}(-1)^{k-i}\left(\begin{array}{c}
k \\
i
\end{array}\right) f(x+i a)
$$

Consequently, the highest $k$ for which $\mathrm{D}_{a}^{(k)} f$ is of interest is $k=p-1$, since $\mathrm{D}_{a}^{(p)} f$ is identically zero, regardless of the function $f$. Since the main operation we will use in this paper will be differentiation $p-1$ times in one direction $a$, with $a=1$ in most cases, we will call it generalized differentiation and use the notation

$$
\begin{aligned}
\mathrm{GD}_{a} f & =\mathrm{D}_{a}^{(p-1)} f \\
\mathrm{GD} f & =\mathrm{D}_{1}^{(p-1)} f .
\end{aligned}
$$

In this case equation (1) becomes simpler:

Lemma 1. For any function $f: \mathbb{F}_{p^{n}} \rightarrow \mathbb{F}_{p^{n}}$ we have

$$
\mathrm{GD}_{a} f(x)=\sum_{i=0}^{p-1} f(x+i a) .
$$

Proof. Use (1) for $k=p-1$ and the fact that in a field of characteristic $p$ we have

$$
\left(\begin{array}{c}
p-1 \\
i
\end{array}\right)=\frac{(p-1)(p-2) \cdots(p-i)}{1 \cdot 2 \cdots i}=\frac{(-1)(-2) \cdots(-i)}{1 \cdot 2 \cdots i}=(-1)^{i}
$$

Note that $\mathrm{GD}_{a} f(x)=\mathrm{GD}_{a} f(x+i a)$ for all $i \in \mathbb{F}_{p}$, so if the equation $\mathrm{GD}_{a} f(x)=b$ has at least one solution, then it has at least $p$ solutions. In view of these results, we can give an alternative definition of GAPN functions:

Proposition 1. A function $f: \mathbb{F}_{p^{n}} \rightarrow \mathbb{F}_{p^{n}}$ is GAPN iff for all $a \in \mathbb{F}_{p^{n}} \backslash\{0\}$, the function $\mathrm{GD}_{a} f(x)$ is $p$-to-1, i.e. for all $b \in \mathbb{F}_{p^{n}}$ the equation $\mathrm{GD}_{a} f(x)=b$ has either 0 or $p$ solutions.

Recall that a function $f: \mathbb{F}_{p^{n}} \rightarrow \mathbb{F}_{p^{n}}$ has a unique representation as an univariate polynomial of degree at most $p^{n}-1$, i.e. $f(x)=\sum_{i=0}^{p^{n}-1} c_{i} x^{i}$ with $c_{i} \in \mathbb{F}_{p^{n}}$. In addition to the degree of this univariate polynomial, we also use the algebraic degree of $f$, denoted $\operatorname{deg}(f)$ defined as follows: if $f(x)=x^{d}$ then the algebraic degree of $f$ is the sum of the digits of $d$ when written in base $p$, i.e. writing $d=\sum_{i=0}^{n-1} d_{i} p^{i}$ with $0 \leq d_{i} \leq p-1$ we have $\operatorname{deg}(f)=\sum_{i=0}^{n-1} d_{i}$. For an arbitrary $f$, its degree is the maximum of the degrees of its monomials.

The affine functions $A: \mathbb{F}_{p^{n}} \rightarrow \mathbb{F}_{p^{n}}$ are the functions of algebraic degree 1, i.e. $A(x)=a+\sum_{i=0}^{n-1} a_{i} x^{p^{i}}$ with $a, a_{i} \in \mathbb{F}_{p^{n}}$. For the particular case $a=0$ these are called linearized polynomials, and they are $\mathbb{F}_{p}$-linear functions.

Two functions $f_{1}, f_{2}$ are called extended affine equivalent (or EA-equivalent) if $f_{1}=A_{1} \circ f_{2} \circ A_{2}+A_{3}$ for some affine functions $A_{1}, A_{2}, A_{3}: \mathbb{F}_{p^{n}} \rightarrow \mathbb{F}_{p^{n}}$ with 
$A_{1}, A_{2}$ invertible. For example, $x^{d}$ is affine equivalent to $x^{p^{i} d} \bmod \left(p^{n}-1\right)$ for any integer $i$ because $x \mapsto x^{p^{i}}$ is an invertible affine function. The APN property is invariant to affine equivalence, and it was shown (see [9, Proposition 2.1] ) that the GAPN property is also invariant to affine equivalence.

The algebraic degree of a function decreases by at least one with each differentiation (see [10]). If $g$ is a function of algebraic degree $p-1$ or less, the function $\mathrm{GD}_{a} g(x)$ is constant. Therefore GAPN functions need to have algebraic degree at least $p$. We introduce a generalization of the affine equivalence, which is more natural in the context of GAPN functions:

Definition 2. Two functions $f_{1}, f_{2}: \mathbb{F}_{p^{n}} \rightarrow \mathbb{F}_{p^{n}}$ are called generalized extended affine equivalent (or GEA-equivalent) if $f_{1}=A_{1} \circ f_{2} \circ A_{2}+g$ for some $g, A_{1}, A_{2}$ : $\mathbb{F}_{p^{n}} \rightarrow \mathbb{F}_{p^{n}}$ with $g$ having algebraic degree at most $p-1$ and $A_{1}, A_{2}$ being invertible affine functions.

The GAPN property is invariant to GEA-equivalence:

Proposition 2. Let $f_{1}, f_{2}: \mathbb{F}_{p^{n}} \rightarrow \mathbb{F}_{p^{n}}$ be GEA-equivalent. Then $f_{1}$ is GAPN iff $f_{2}$ is GAPN.

When searching for GAPN functions, we therefore need only consider functions up to GEA-equivalence. Note that for $p=2$ the GEA-equivalence coincides with the usual EA-equivalence, but for $p>2$ the GEA-equivalence is coarser than EA-equivalence. In other words, a GAPN function which appears to be new when testing for EA-equivalence with existing functions, might in fact not be new if we test for GEA-equivalence.

In order to search for GAPN functions among the monomial functions, we recall the following results:

Lemma 2. (see [16]) Let $f: \mathbb{F}_{p^{n}} \rightarrow \mathbb{F}_{p^{n}}, f(x)=x^{d}$ and $a \in \mathbb{F}_{p^{n}}, a \neq 0$. We have

$$
\mathrm{GD}_{a} f(x)=a^{d} \operatorname{GD} f(x / a) .
$$

Consequently, $f$ is GAPN iff GD $f$ is p-to-1.

Using Lucas's theorem and the fact that in a field of characteristic $p$ we have that $\sum_{i \in \mathbb{F}_{p}} i^{d}$ equals -1 if $d$ is a multiple of $p-1$, and it equals 0 otherwise, we have:

Lemma 3. (see [16]) Let $f: \mathbb{F}_{p^{n}} \rightarrow \mathbb{F}_{p^{n}}, f(x)=x^{d}$. Write $d$ in base $p$ as $d=\sum_{i=0}^{n-1} d_{i} p^{i}$ with $0 \leq d_{i} \leq p-1$. We have

$$
\begin{aligned}
\operatorname{GD} f(x) & =-\sum_{\substack{1 \leq j \leq d \\
(p-1) \mid j}}\left(\begin{array}{l}
d \\
j
\end{array}\right) x^{d-j} \\
& =-\sum_{\substack{j=\sum_{i=0}^{n-1} j_{i} p^{i} \neq 0 \\
(p-1) \mid j}}\left(\begin{array}{c}
d_{0} \\
j_{0}
\end{array}\right) \cdots\left(\begin{array}{c}
d_{n-1} \\
j_{n-1}
\end{array}\right) x^{d-j}
\end{aligned}
$$

where $0 \leq j_{i} \leq d_{i}$ for $i=0, \ldots, n-1$. 
Monomial functions of algebraic degree $p$ are described in [9, Lemma 3.3] and $[8$, Theorem 2.2]. The condition we give below produces the same class of functions, but has the advantage of being easier to check algorithmically.

Theorem 1. Let $f: \mathbb{F}_{p^{n}} \rightarrow \mathbb{F}_{p^{n}}, f(x)=x^{d}$ where $d=\sum_{i=1}^{s} k_{i} p^{\ell_{i}}$, with $0 \leq$ $\ell_{1}<\ell_{2}<\ldots<\ell_{s}, 0 \leq k_{i} \leq p-1$. Assume $f$ has algebraic degree $p$, i.e. $\sum_{i=1}^{s} k_{i}=p$.

(i) We have

$$
\operatorname{GD} f(x)=-\sum_{i=1}^{s} k_{i} x^{p^{\ell_{i}}}
$$

(ii) $f$ is a GAPN function iff $\operatorname{gcd}\left(\sum_{i=1}^{s} k_{i} z^{\ell_{i}}, z^{n}-1\right)=z-1$ in the polynomial $\operatorname{ring} \mathbb{F}_{p}[z]$.

Proof. For (i) we will use Lemma 3 above:

$$
\operatorname{GD} f(x)=-\sum\left(\begin{array}{c}
k_{1} \\
j_{1}
\end{array}\right) \cdots\left(\begin{array}{c}
k_{s} \\
j_{s}
\end{array}\right) x^{\sum_{i=1}^{s}\left(k_{i}-j_{i}\right) p^{\ell_{i}}}
$$

where the sum is over all $\left(j_{1}, \ldots, j_{s}\right)$ such that $0 \leq j_{i} \leq k_{i}$ and $j=\sum_{i=1}^{s} j_{i} p^{\ell_{i}}$ is non-zero and divisible by $p-1$. Consider such a tuple $\left(j_{1}, \ldots, j_{s}\right)$. The value $j$ is divisible by $p-1$ iff the sum of its digits, i.e. $\sum_{i=1}^{s} j_{i}$, is divisible by $p-1$. Since $0<\sum_{i=1}^{s} j_{i} \leq \sum_{i=1}^{s} k_{i}=p$, we must have $\sum_{i=1}^{s} j_{i}=p-1$. Therefore all digits of $j$ must be equal to the corresponding digits of $d$ (in which case $\left(\begin{array}{l}k_{i} \\ j_{i}\end{array}\right)=\left(\begin{array}{l}k_{i} \\ k_{i}\end{array}\right)=1$ ), except for one digit of $j$, which should be one less than the corresponding digit of $d$ (in which case $\left.\left(\begin{array}{c}k_{i} \\ j_{i}\end{array}\right)=\left(\begin{array}{c}k_{i} \\ k_{i}-1\end{array}\right)=k_{i}\right)$. Substituting these values in (3) we obtain (2).

For (ii) let $\psi: \mathbb{F}_{p^{n}} \rightarrow \mathbb{F}_{p^{n}}$ be the $\mathbb{F}_{p^{-}}$linear map defined as $\psi(x)=\operatorname{GD} f(x)$. Hence $f$ is GAPN iff $\operatorname{dim}_{\mathbb{F}_{p}} \operatorname{Ker} \psi=1$. Let $M(x) \in \mathbb{F}_{p}[x]$ be the polynomial given by $M(x)=\operatorname{gcd}\left(\sum_{i=1}^{s} k_{i} x^{p^{\ell_{i}}}, x^{p^{n}}-x\right)$. Note that $M(x)$ splits into its linear factors over $\mathbb{F}_{p^{n}}$ and Ker $\psi$ is exactly the set consisting of the roots of $M(x)$. Let $m(z) \in \mathbb{F}_{p}[z]$ be the polynomial given by $m(z)=\operatorname{gcd}\left(\sum_{i=1}^{s} k_{i} z^{\ell_{i}}, z^{n}-1\right)$. As $M(x) \in \mathbb{F}_{p}[x]$ and $M(x)$ is a linearized polynomial it follows from [11, Theorem 3.62] that $\operatorname{dim}_{\mathbb{F}_{p}} \operatorname{Ker} \psi=1$ iff $\operatorname{deg}(m(z))=1$. Moreover $(z-1) \mid m(z)$ as the evaluation of $m(z)$ at $z=1$ is $\sum_{i=1}^{s} k_{i}=p=0 \bmod p$. This completes the proof.

\section{GAPN functions of the form $x^{k_{2} p^{\ell_{2}}+k_{1} p^{\ell_{1}}}$ (Generalized Gold functions)}

In this section we examine monomial functions $f(x)=x^{d}$ with $d$ such that it contains only two non-zero digits when written in base $p$. Some GAPN functions of this type were presented in [9] and [16]. We determine further exponents $d$ which produce GAPN functions.

We will need the following lemma: 
Lemma 4. Let $b \in \mathbb{F}_{p^{n}}$ and let $\ell$ be an integer coprime to $n$, with $1 \leq \ell<n$. (i) The equation $x^{p^{\ell}}-x=b$ has solutions iff $\operatorname{Tr}_{n / 1}(b)=0$, where $\operatorname{Tr}_{n / 1}($ ) denotes the absolute trace.

(ii) If the equation $x^{p^{e}}-x=b$ has a solution $x_{1}$ then it has exactly $p$ solutions, namely $x_{1}+i, i \in \mathbb{F}_{p}$.

Proof. (i) Let $\psi: \mathbb{F}_{p^{n}} \rightarrow \mathbb{F}_{p^{n}}$ be the additive homomorphism given by $\psi(x)=$ $x^{p^{\ell}}-x$. Note that $x \in \operatorname{Ker} \psi$ iff $x^{p^{\ell}}=x$. Since $x^{p^{n}}=x$ for all $x \in \mathbb{F}_{p^{n}}$, we have that $x \in \operatorname{Ker} \psi$ is equivalent to $x=x^{\operatorname{gcd}\left(p^{n}, p^{\ell}\right)}=x^{p^{\operatorname{gcd}(n, \ell)}}=x^{p}$, hence Ker $\psi=\mathbb{F}_{p}$. If $b=\psi(x)$ for some $x \in \mathbb{F}_{p^{n}}$, then $\operatorname{Tr}_{n / 1}(b)=b+b^{p}+\cdots+$ $b^{p^{n-1}}=\left(x+x^{p}+\cdots+x^{p^{n-1}}\right)^{p^{\ell}}-\left(x+x^{p}+\cdots+x^{p^{n-1}}\right)=0$. As $\psi$ is linear and $\operatorname{dim}_{\mathbb{F}_{p}} \operatorname{Ker} \psi=1$, we must have $\operatorname{dim}_{\mathbb{F}_{p}} \operatorname{Im} \psi=n-1$. Since we showed that the image of $\psi$ is included in the set of elements of trace zero, which is a space of dimension $n-1$, we conclude that for all $b \in \mathbb{F}_{p^{n}}$, there exists $x \in \mathbb{F}_{p^{n}}$ such that $b=\psi(x)$ iff $\operatorname{Tr}_{n / 1}(b)=0$.

(ii) It is easy to check that $x_{1}+i, i \in \mathbb{F}_{p}$ are indeed solutions. To check whether the equation has more than $p$ solutions, let $x_{1}, x_{2}$ be two different solutions. That means $x_{1}^{p^{\ell}}-x_{1}=x_{2}^{p^{\ell}}-x_{2}$, i.e. $\left(x_{1}-x_{2}\right)^{p^{\ell}}=\left(x_{1}-x_{2}\right)$. This equation holds iff the order of $x_{1}-x_{2}$ is a factor of $p^{\ell}-1$; since the orders of all elements of $\mathbb{F}_{p^{n}}$ are factors of $p^{n}-1$, we have that the order of $x_{1}-x_{2}$ is a factor of $\operatorname{gcd}\left(p^{\ell}-1, p^{n}-1\right)=p^{\operatorname{gcd}(\ell, n)}-1=p-1$. The only elements which have order a factor of $p-1$ are the elements of $\mathbb{F}_{p}$, i.e. $x_{1}-x_{2} \in \mathbb{F}_{p}$, so there are exactly $p$ distinct solutions.

Theorem 2. Let $f: \mathbb{F}_{p^{n}} \rightarrow \mathbb{F}_{p^{n}}, f(x)=x^{d}$ where $d=k_{2} p^{\ell_{2}}+k_{1} p^{\ell_{1}}$, with $\ell_{1}<\ell_{2}, 0 \leq k_{1}, k_{2} \leq p-1$ and $p \leq k_{1}+k_{2}<2(p-1)$.

(i) We have

$$
\operatorname{GD} f(x)=-\left(\begin{array}{c}
k_{2} \\
u
\end{array}\right)\left(x^{p^{\ell_{2}}}-x^{p^{\ell_{1}}}\right)^{u}
$$

where $u=k_{1}+k_{2}-(p-1)$.

(ii) If $\operatorname{gcd}\left(\ell_{2}-\ell_{1}, n\right)=1$ and $\operatorname{gcd}\left(u, p^{n}-1\right)=1$ then $f$ is a GAPN function.

(iii) If $f$ is a GAPN function then $\operatorname{gcd}\left(\ell_{2}-\ell_{1}, n\right)=1$ and $\operatorname{gcd}\left(u, p^{n_{1}}-1\right)=1$ for all values of $n_{1}$ such that $n_{1} \mid n$ and $n_{1}<n$.

(iv) The converses of (ii) and (iii) do not hold. More precisely, there are both $G A P N$ functions and non-GAPN functions among the functions with $\operatorname{gcd}\left(\ell_{2}-\right.$ $\left.\ell_{1}, n\right)=1$ and $\operatorname{gcd}\left(u, p^{n_{1}}-1\right)=1$ for all values of $n_{1}$ such that $n_{1} \mid n$ and $n_{1}<n$, but $\operatorname{gcd}\left(u, p^{n}-1\right)>1$.

Proof. We will prove the theorem for the case $\ell_{1}=0$. For the general case we do first the invertible affine transformation $A$ defined by $x \mapsto x^{p^{n-\ell_{1}}}$, which makes the function become $(f \circ A)(x)=x^{k_{2} p^{\ell_{2}-\ell_{1}}+k_{1}}$.

For (i), the proof generalizes the proof of [16, Theorem 2.4]. Lemma 3 implies:

$$
\operatorname{GD} f(x)=-\sum\left(\begin{array}{c}
k_{2} \\
j_{2}
\end{array}\right)\left(\begin{array}{c}
k_{1} \\
j_{1}
\end{array}\right) x^{\left(k_{2}-j_{2}\right) p^{\ell_{2}}+\left(k_{1}-j_{1}\right)}
$$


where the sum is over all $j_{1}, j_{2}$ such that $0 \leq j_{1} \leq k_{1}, 0 \leq j_{2} \leq k_{2}$ and $j=j_{2} p^{\ell_{2}}+j_{1}$ is non-zero and is divisible by $p-1$. A number written in base $p$ is divisible by $p-1$ iff the sum of its digits is divisible by $p-1$. Hence $j_{1}+j_{2}$ is a multiple of $p-1$. From the assumptions in the theorem statement, $k_{1}$ and $k_{2}$ are not both equal to $p-1$. Therefore we must have $j_{1}+j_{2}=p-1$. The equation above becomes

$$
\operatorname{GD} f(x)=-\sum_{j_{2}=p-1-k_{1}}^{k_{2}}\left(\begin{array}{c}
k_{2} \\
j_{2}
\end{array}\right)\left(\begin{array}{c}
k_{1} \\
p-1-j_{2}
\end{array}\right) x^{\left(k_{2}-j_{2}\right) p^{\ell_{2}}+\left(k_{1}-(p-1)+j_{2}\right)}
$$

We aim to re-arrange the product of two combinatorial coefficients in the sum above so that the variable of summation $j_{2}$ only appears in one of them rather than in both. The following known combinatorial identity can help:

$$
\left(\begin{array}{c}
m \\
k
\end{array}\right)\left(\begin{array}{l}
k \\
h
\end{array}\right)=\left(\begin{array}{c}
m \\
h
\end{array}\right)\left(\begin{array}{l}
m-h \\
m-k
\end{array}\right)
$$

but in order to use it, we first need to "swap" the top and bottom part of a combinatorial coefficient using the following identity which holds in fields of characteristic $p$, and which can be proven by using the fact that $m !=(-1)^{m}(p-$ $1) ! /(p-1-m) !$

$$
\left(\begin{array}{l}
m \\
k
\end{array}\right)=\frac{m !}{k !(m-k) !}=\frac{(-1)^{m}(p-1) !(p-1-k) !}{(-1)^{k}(p-1) !(p-1-m) !(m-k) !}=(-1)^{m-k}\left(\begin{array}{c}
p-1-k \\
p-1-m
\end{array}\right) .
$$

So in our situation, applying (6), then (5) and then the usual $\left(\begin{array}{c}m \\ k\end{array}\right)=\left(\begin{array}{c}m \\ m-k\end{array}\right)$ we have:

$$
\begin{aligned}
\left(\begin{array}{c}
k_{2} \\
j_{2}
\end{array}\right)\left(\begin{array}{c}
k_{1} \\
p-1-j_{2}
\end{array}\right) & =(-1)^{j_{2}+k_{1}-(p-1)}\left(\begin{array}{c}
k_{2} \\
j_{2}
\end{array}\right)\left(\begin{array}{c}
j_{2} \\
p-1-k_{1}
\end{array}\right) \\
& =(-1)^{j_{2}+k_{1}-(p-1)}\left(\begin{array}{c}
k_{2} \\
p-1-k_{1}
\end{array}\right)\left(\begin{array}{c}
k_{1}+k_{2}-(p-1) \\
k_{2}-j_{2}
\end{array}\right) \\
& =(-1)^{j_{2}+k_{1}-(p-1)}\left(\begin{array}{c}
k_{2} \\
k_{1}+k_{2}-(p-1)
\end{array}\right)\left(\begin{array}{c}
k_{1}+k_{2}-(p-1) \\
j_{2}+k_{1}-(p-1)
\end{array}\right) .
\end{aligned}
$$

We use this in equation (4), then use a new index of summation $i=j_{2}+k_{1}-$ $(p-1)$ :

$$
\begin{aligned}
\operatorname{GD} f(x) & =-\left(\begin{array}{c}
k_{2} \\
u
\end{array}\right) \sum_{j_{2}=p-1-k_{1}}^{k_{2}}\left(\begin{array}{c}
u \\
j_{2}+k_{1}-(p-1)
\end{array}\right) x^{\left(k_{2}-j_{2}\right) p^{\ell_{2}}+\left(j_{2}+k_{1}-(p-1)\right)}(-1)^{j_{2}+k_{1}-(p-1)} \\
& =-\left(\begin{array}{c}
k_{2} \\
u
\end{array}\right) \sum_{i=0}^{u}\left(\begin{array}{c}
u \\
i
\end{array}\right) x^{(u-i) p^{\ell_{2}}}(-x)^{i} \\
& =-\left(\begin{array}{c}
k_{2} \\
u
\end{array}\right)\left(x^{p^{\ell_{2}}}-x\right)^{u} .
\end{aligned}
$$


For (ii), by Lemma 2 we only need to prove that GD $f$ is $p$-to- 1 . Let $b \in \mathbb{F}_{p^{n}}$ and consider the equation $\operatorname{GD} f(x)=b$, i.e. $-\left(\begin{array}{c}k_{2} \\ u\end{array}\right)\left(x^{p^{\ell_{2}}}-x\right)^{u}=b$. One can check that $\left(\begin{array}{c}k_{2} \\ u\end{array}\right) \neq 0$, so we have $\left(x^{p^{\ell_{2}}}-x\right)^{u}=-b\left(\begin{array}{c}k_{2} \\ u\end{array}\right)^{-1}$. Since $\operatorname{gcd}\left(u, p^{n}-1\right)=1$, $u$ is invertible modulo $p^{n}-1$, so let $u^{\prime}$ be its inverse. The equation becomes $x^{p^{\ell_{2}}-x}=\left(-b\left(\begin{array}{c}k_{2} \\ u\end{array}\right)^{-1}\right)^{u^{\prime}}$. By Lemma 4(ii), this equation has either 0 or $p$ solutions.

Next we prove (iii). Assume first that $\operatorname{gcd}\left(\ell_{2}-\ell_{1}, n\right)>1$. Put $v=\operatorname{gcd}\left(\ell_{2}-\right.$ $\left.\ell_{1}, n\right)$. Consider the intermediate field $\mathbb{F}_{p} \subsetneq \mathbb{F}_{p^{v}} \subseteq \mathbb{F}_{p^{n}}$. For any $\alpha \in \mathbb{F}_{p^{v}}$ we have $\alpha^{p^{v}}-\alpha=0$ and hence $\operatorname{GD} f(\alpha)=0$. Therefore $f$ is not a GAPN function as $p^{v}>p$.

Next we assume that $\operatorname{gcd}\left(\ell_{2}-\ell_{1}, n\right)=1$ and $\operatorname{gcd}\left(k_{1}+k_{2}-(p-1), p^{n_{1}}-1\right)>1$ for a positive divisor $n_{1} \mid n$ such that $n_{1}<n$. We prove that $f$ is not a GAPN function.

Consider the multiplicative homomorphism $\varphi: \mathbb{F}_{p^{n_{1}}}^{*} \rightarrow \mathbb{F}_{p^{n_{1}}}^{*}$ given by $\varphi(x)=$ $x^{u}$. Note that $\operatorname{Ker} \varphi=\left\{x \in \mathbb{F}_{p^{n_{1}}}: x^{u}=1\right\}=\left\{x \in \mathbb{F}_{p^{n_{1}}}: x^{\operatorname{gcd}\left(u, p^{n_{1}}-1\right)}=1\right\}$. Hence the cardinality of $\operatorname{Ker} \varphi$ is $\operatorname{gcd}\left(u, p^{n_{1}}-1\right)>1$ and there exists

$$
\mu \in \mathbb{F}_{p^{n_{1}}}^{*} \backslash\{1\} \text { such that } \mu^{u}=1 .
$$

Let $\operatorname{Tr}_{n / n_{1}}$ and $\operatorname{Tr}_{n / 1}$ denote the trace maps from $\mathbb{F}_{p^{n}}$ onto $\mathbb{F}_{p^{n_{1}}}$ and $\mathbb{F}_{p}$, respectively. Similarly let $\operatorname{Tr}_{n_{1} / 1}$ denote the trace map from $\mathbb{F}_{p^{n_{1}}}$ onto $\mathbb{F}_{p}$. Recall that

$$
\operatorname{Tr}_{n / 1}=\operatorname{Tr}_{n_{1} / 1} \circ \operatorname{Tr}_{n / n_{1}}
$$

as a composition of maps.

Let $y_{1} \in \mathbb{F}_{p^{n}}^{*}$ such that $\operatorname{Tr}_{n / n_{1}}(y)=0$, which exists as $n_{1} \mid n$ and $n_{1}<n$. Put $\beta=y_{1}^{u} \in \mathbb{F}_{p^{n}}^{*}$. We will show that the equation

$$
\left(x^{p^{\ell_{2}}}-x^{p^{\ell_{1}}}\right)^{u}=\beta^{p^{\ell_{1}}}
$$

has at least $2 p$ solutions, which completes the proof. Note that this is equivalent to show that the equation

$$
\left(x^{p^{\ell_{2}-\ell_{1}}}-x\right)^{u}=\beta
$$

has at least $2 p$ solutions. First consider the set

$$
S_{1}=\left\{x \in \mathbb{F}_{p^{n}}: x^{p^{\ell_{2}-\ell_{1}}}-x=y_{1}\right\} .
$$

From Lemma 4(ii) the cardinality of $S_{1}$ is either 0 or $p$. Moreover the cardinality of $S_{1}$ is $p$ iff $y_{1}=\psi(x)$ for some $x \in \mathbb{F}_{p^{n}}$, where $\psi$ is the additive homomorphism defined as $\psi(x)=x^{p^{\ell_{2}-\ell_{1}}}-x$. Using (8) and the fact that $\operatorname{Tr}_{n / n_{1}}\left(y_{1}\right)=0$ by the choice of $y_{1}$, we have that $\operatorname{Tr}_{n / 1}\left(y_{1}\right)=\operatorname{Tr}_{n_{1} / 1} \circ \operatorname{Tr}_{n / n_{1}}\left(y_{1}\right)=0$. By Lemma 4 we conclude that the cardinality of $S_{1}$ is $p$.

Next put $y_{2}=\mu y_{1} \in \mathbb{F}_{p^{n}}^{*}$, where $\mu$ is chosen as in (7). As $y_{1} \neq 0$ and $\mu \neq 1$ we know that $y_{2} \neq y_{1}$. Consider the set

$$
S_{2}=\left\{x \in \mathbb{F}_{p^{n}}: x^{p^{\ell_{2}-\ell_{1}}}-x=y_{2}\right\} .
$$


As $y_{2} \neq y_{1}$, it is clear that $S_{1} \cap S_{2}=\emptyset$. Moreover $\operatorname{Tr}_{n / n_{1}}\left(y_{2}\right)=\operatorname{Tr}_{n / n_{1}}\left(\mu y_{1}\right)=$ $\mu \operatorname{Tr}_{n / n_{1}}\left(y_{1}\right)=0$, where we use the fact that $\mu \in \mathbb{F}_{p^{n_{1}}}$. Hence following the same arguments we used for the cardinality of $S_{1}$ above we obtain that the cardinality of $S_{2}$ is $p$ as well. Therefore $\left|S_{1} \cup S_{2}\right|=2 p$. Now we complete the proof by showing that if $x \in S_{1} \cup S_{2}$, then $x$ satisfies (9). Indeed if $x \in S_{1}$, then

$$
\left(x^{p^{\ell_{2}-\ell_{1}}}-x\right)^{u}=y_{1}^{u}=\beta
$$

by definition of $\beta$. Similarly if $x \in S_{2}$, then

$$
\left(x^{p^{\ell_{2}-\ell_{1}}}-x\right)^{u}=y_{2}^{u}=\left(\mu y_{1}\right)^{u}=\mu^{u} y_{1}^{u}=\beta
$$

as $\mu^{u}=1$ by the choice of $\mu$ in (7).

(iv) We consider the monomial functions $x^{d}$ with $\operatorname{gcd}\left(\ell_{2}-\ell_{1}, n\right)=1$ and:

$$
\begin{aligned}
& -p=5, n=2, k_{1}+k_{2}=7 \text { (i.e. } u=3 \text { ) } \\
& -p=11, n=2, k_{1}+k_{2}=13 \text { (i.e. } u=3 \text { ) } \\
& -p=7, n=4, k_{1}+k_{2}=11 \text { (i.e. } u=5 \text { ). }
\end{aligned}
$$

For all these cases one can easily check that $\operatorname{gcd}\left(u, p^{n}-1\right)>1$ but $\operatorname{gcd}\left(u, p^{n_{1}}-\right.$ 1) $=1$ for all values of $n_{1}$ such that $n_{1} \mid n$ and $n_{1}<n$.

Computing $\operatorname{GD} f(x)$ using (i) and then exhaustively evaluating it, by computer, at all $x \in \mathbb{F}_{p^{n}}$ to decide whether it is $p$-to- 1 , one can check that the first and second cases above produce GAPN functions whereas the third one does not.

For $p=2$, Theorem 2 above states that $x^{2^{\ell_{2}}+2^{\ell_{1}}}$ is APN over $\mathbb{F}_{2^{n}}$ iff $\operatorname{gcd}\left(\ell_{2}-\right.$ $\left.\ell_{1}, n\right)=1$, i.e. we retrieve the known result which states that Gold functions are APN. We could therefore call the functions satisfying Theorem 2(ii) Generalized Gold functions (note that they are more general than those called Generalized Gold functions by Kuroda and Tsujie in [9, Theorem 3.4], which would correspond to the particular case $k_{2}=1$ and $k_{1}=p-1$ ).

The first two classes of GAPN functions described by Zha et. al. in [16, Theorems 2.4 and 3.1] can also be obtained as particular cases of Theorem 2 above; namely putting $\ell_{2}=1, \ell_{1}=0$ we obtain their first class of functions and putting $\ell_{1}=0$ and $k_{1}=p-1$ we obtain their second class.

One can show that for any integer $u$, if $\operatorname{gcd}(u, p-1)=1$ then $\operatorname{gcd}\left(u, p^{n}-1\right)=1$ for infinitely many values of $n$. (Namely, let $p_{1}, \ldots, p_{s}$ be the prime factors of $u$ and let $n_{i}$ be the lowest integers such that $p_{i} \mid p^{n_{i}}-1$, i.e. $n_{i}$ is the order of $p$ modulo $p_{i}$. Then $\operatorname{gcd}\left(u, p^{n}-1\right)>1$ iff there is an $i$ such that $p_{i} \mid p^{n}-1$ iff there is an $i$ such that $n_{i} \mid n$. Hence for all $n$ except those divisible by some $n_{i}$ we have $\operatorname{gcd}\left(u, p^{n}-1\right)=1$ ) Therefore, by Theorem 2(ii), any function $f=x^{d}$ with $d=k_{2} p^{\ell_{2}}+k_{1} p^{\ell_{1}}$, with $\ell_{1}<\ell_{2}, \operatorname{gcd}\left(\ell_{2}-\ell_{1}, n\right)=1,0 \leq k_{1}, k_{2} \leq p-1$, $p \leq k_{1}+k_{2}$, and $\operatorname{gcd}\left(k_{1}+k_{2}, p-1\right)=1$ is GAPN as a function over $\mathbb{F}_{p^{n}}$ for infinitely many $n$. The exponents with this property are called $p$-exceptional in $[8$, Definition 1.1]. We have therefore fully disproved [8, Conjecture 2.7] which states that for algebraic degrees between $p+1$ and $n(p-1)-2$ there are no 
monomial GAPN functions and no $p$-exceptional exponents. In fact the classes of functions given in [16] already disprove this conjecture fully, but the authors only realized that they disproved the first part of the conjecture.

Example 1. Consider the field $\mathbb{F}_{11^{5}}$ and the functions $f=x^{d}$ with $d=4 \cdot 11^{2}+9$ or $d=8 \cdot 11^{2}+9$. We have $\ell_{2}-\ell_{1}=2-0$ which is coprime to $n=5$. Also $u=4+9-(11-1)=3$ in the first case, and $u=8+9-(11-1)=7$ in the second case, both of which are relatively prime to $11^{5}-1=161050$. By Theorem 2(ii), these are GAPN functions. Note that these functions cannot be obtained by the constructions in previous work ([9], [16]).

\section{Connection between GAPN functions and certain constructions of permutations}

In [6], Hou has shown that for each integer $d \geq 1$ and $q$ a power of $p$, there exists a unique polynomial in $g_{d, q}(y) \in \mathbb{F}_{p}[y]$ with the property:

$$
g_{d, q}\left(x^{p}-x\right)=\sum_{u \in \mathbb{F}_{q}}(x+u)^{d}
$$

In [7], Hou explores ways to find permutation polynomials over $\mathbb{F}_{q^{n}}$, for different values of $n$, among the polynomial functions defined by $g_{d, q}$.

Here we will only consider the case $q=p$. Using Lemma 1, we see that in this case $g_{d, p}\left(x^{p}-x\right)=\operatorname{GD} f(x)$, where $f(x)=x^{d}$. The situations when $g_{d, q}$ is a permutation lead to constructing GAPN functions:

Theorem 3. Let $d$ be a positive integer and $f: \mathbb{F}_{p^{n}} \rightarrow \mathbb{F}_{p^{n}}$ be the function $f(x)=x^{d}$. Let $g_{d, q}$ be the function defined by (10). If $g_{d, q}$ is a permutation on $\mathbb{F}_{p^{n}}$ then $f$ is a GAPN function.

Proof. Let $b \in \mathbb{F}_{p^{n}}$ and consider the equation $\operatorname{GD} f(x)=b$. This is equivalent to $g_{d, p}\left(x^{p}-x\right)=b$. Since $g_{d, p}$ is a permutation, there is a unique element $c \in \mathbb{F}_{p^{n}}$ such that $g_{d, p}(c)=b$. The solutions of the equation $g_{d, p}\left(x^{p}-x\right)=b$ are exactly the solutions of the equation $x^{p}-x=c$, which has at most $p$ solutions. Hence $\operatorname{GD} f(x)$ is $p$-to- 1 and by Lemma $2 f$ is GAPN.

The converse of the Theorem above does not hold, i.e. there are GAPN functions of the form $f(x)=x^{d}$ for which $g_{d, q}$ is not a permutation, see Example 2 below.

The Theorem above has the potential of constructing new GAPN functions. For $p=3$, Hou lists in [7, Table 2 and 3] some values $d$ and $n$ (called in those tables $n$ and $e$ ) for which $g_{d, p}$ is a permutation over $\mathbb{F}_{p^{n}}$. In view of Theorem 3 , for all these values $d$ and $n$ we have that $x^{d}$ is a GAPN function over $\mathbb{F}_{p^{n}}$. We examined all these values, and they produce no new functions; all functions are in the classes of functions described by Theorem 1 and Theorem 2 . 
Remark 1. Theorem 3 also gives an alternative proof of Theorem 2(ii) for the case when $\ell_{2}-\ell_{1}$ is not divisible by $p$. Namely, we can write $\operatorname{GD} f(x)$ as a function in $x^{p}-x$ :

$$
\operatorname{GD} f(x)=-\left(\begin{array}{c}
k_{2} \\
u
\end{array}\right)\left(\left(x^{p}-x\right)^{p^{\ell_{2}-1}}+\left(x^{p}-x\right)^{p^{\ell_{2}-2}}+\ldots\left(x^{p}-x\right)^{p^{\ell_{1}}}\right)^{u}
$$

i.e. $\operatorname{GD} f(x)=g_{d, p}\left(x^{p}-x\right)$ where $g_{d, p}(y)=-\left(\begin{array}{c}k_{2} \\ u\end{array}\right) h(y)^{u}$ with $h(y)=y^{p^{\ell_{2}-1}}+$ $\ldots+y^{p^{\ell_{1}}}$. This function $h(y)^{u}$ is a permutation if $\operatorname{gcd}\left(u, p^{n}-1\right)=1$ and $h(y)$ is a permutation. Using [11, Theorem 3.62] similar to the proof of Theorem 1(ii), we see that $h(y)$ is a permutation iff $\operatorname{gcd}\left(z^{\ell_{2}-1}+z^{\ell_{2}-2}+\ldots z^{\ell_{1}}, z^{n}-1\right)=1$ in the polynomial ring $\mathbb{F}_{p}[z]$, which holds iff $\operatorname{gcd}\left(\ell_{2}-\ell_{1}, n\right)=1$ (here we used the assumption that $\ell_{2}-\ell_{1}$ not divisible by $p$ ).

Even when $g_{d, q}$ is not a permutation, there are still cases where it can be used for constructing GAPN functions:

Theorem 4. Let $f, \psi: \mathbb{F}_{p^{n}} \rightarrow \mathbb{F}_{p^{n}}$ be the functions defined as $f(x)=x^{d}$ for a positive integer $d$ and $\psi(x)=x^{p}-x$. Let $k \geq 1$ be the highest integer such that there is a polynomial $h_{d, p}(y)$ in $\mathbb{F}_{p}[y]$ such that $\operatorname{GD} f=h_{d, p} \circ \psi^{k}$ as functions over $\mathbb{F}_{p^{n}}$ (where $\psi^{k}=\psi \circ \psi \cdots \circ \psi$ ). If $n$ is not divisible by $p$ then

(i) $f$ is a GAPN function over $\mathbb{F}_{p^{n}}$ iff for any $b \in \mathbb{F}_{p^{n}}$ there is at most one element with trace zero in the preimage of $b$ under $h_{d, p}$, i.e.

$$
\left|\left\{y \in \mathbb{F}_{p^{n}}: h_{d, p}(y)=b, \operatorname{Tr}_{n / 1}(y)=0\right\}\right| \leq 1 .
$$

(ii) If $h_{d, p}$ is a permutation of $\mathbb{F}_{p^{n}}$, then $x^{d}$ is a GAPN function over $\mathbb{F}_{p^{n}}$.

To prove this Theorem we will use the following Lemma, related to Lemma 4.

Lemma 5. Let $\psi: \mathbb{F}_{p^{n}} \rightarrow \mathbb{F}_{p^{n}}$ defined by $\psi(x)=x^{p}-x$ and denote $\psi^{k}=$ $\psi \circ \psi \circ \cdots \circ \psi$. If $p$ does not divide $n$, then for all $k \geq 1$ we have:

(i) $\psi^{k}$ is an $\mathbb{F}_{p}$ - linear function.

(ii) $\operatorname{Ker} \psi^{k}=\mathbb{F}_{p}$.

(iii) $\operatorname{Tr}_{n / 1} \circ \psi^{k}=0$ where $\operatorname{Tr}_{n / 1}$ is the trace function.

(iv) $\psi^{k}$ is a $p$-to-1 function; if $\psi^{k}=b$ does have a solution then it has exactly $p$ solutions, and they are of the form $x_{1}+i, i \in \mathbb{F}_{p}$.

(v) For any $b \in \mathbb{F}_{p^{n}}$ the equation $\psi^{k}(x)=b$ has a solution iff $\operatorname{Tr}_{n / 1}(b)=0$.

Proof. For (i), it is easy to check that $\psi$ is linear, and the composition of linear functions is linear, so $\psi^{k}$ is linear.

For (iii), $\operatorname{Tr}_{n / 1} \circ \psi(x)=\operatorname{Tr}_{n / 1}\left(x^{p}-x\right)=T r_{n / 1}\left(x^{p}\right)-T r_{n / 1}(x)=0$.

For (ii), the inclusion $\mathbb{F}_{p} \subseteq \operatorname{Ker} \psi^{k}$ is obvious, as $\psi(x)=0$ for all $x \in \mathbb{F}_{p}$. We prove the reverse inclusion by induction on $k$. For the base case $k=1$ we have $\psi(x)=0$ iff $x^{p}=x$ iff $x \in \mathbb{F}_{p}$. For the inductive step, assume the statement is true for $k-1$ and we prove it for $k$. Let $x \in \operatorname{Ker} \psi^{k}$ i.e. $\psi^{k}(x)=\psi^{k-1}(\psi(x))=0$. This means $\psi(x) \in \operatorname{Ker} \psi^{k-1}$, and by the induction hypothesis $\operatorname{Ker} \psi^{k-1} \subseteq \mathbb{F}_{p}$. 
Since $\psi(x) \in \mathbb{F}_{p}$, we have $\operatorname{Tr}_{n / 1}(\psi(x))=n \psi(x)$. On the other hand, by (iii) $\operatorname{Tr}_{n / 1}(\psi(x))=0$. If $n$ is not divisible by $p$, then $n \psi(x)=0$ iff $\psi(x)=0$, which happens iff $x \in \mathbb{F}_{p}$.

For (iv), we use the fact that $\psi^{k}$ is $\mathbb{F}_{p^{-}}$-linear, from (i), and its kernel has dimension 1, from (ii).

Finally, for (v), from (iii) we know that $\operatorname{Im} \psi^{k} \subseteq \operatorname{Ker} T r_{n / 1}$. On the other hand by (ii), Ker $\psi^{k}$ has dimension 1 , therefore $\operatorname{Im} \psi^{k}$ has dimension $n-1$. Since Ker $\operatorname{Tr}_{n / 1}$ also has dimension $n-1$, we must have $\operatorname{Im} \psi^{k}=\operatorname{Ker} T r_{n / 1}$.

Proof. (of Theorem 4) (i) For the direct implication assume $f$ is GAPN, and assume for a contradiction that there is an element $b \in \mathbb{F}_{p^{n}}$ for which there are two distinct elements $y_{1}, y_{2}$, both having trace zero and $h_{d, p}\left(y_{1}\right)=h_{d, p}\left(y_{2}\right)=$ $b$. The two equations $\psi^{k}(x)=y_{1}$ and $\psi^{k}(x)=y_{2}$ have $p$ solutions each (see Lemma 5) and the two sets of $p$ solutions are disjoint. These $2 p$ solutions are also solutions to the equation $\operatorname{GD} f(x)=b$. But this contradicts the fact that GD $f$ is a $p$-to- 1 function.

For the reverse implication, let $b \in \mathbb{F}_{p^{n}}$ and consider the equation $\operatorname{GD} f(x)=$ $b$, or equivalently, $h_{d, p}\left(\psi^{k}(x)\right)=b$. The set of solutions to this equation equals to the union of the sets of solutions to the equations $\psi^{k}(x)=y_{1}$, where $y_{1} \in \mathbb{F}_{p^{n}}$ ranges over all the solution to the equation $h_{d, p}(y)=b$. By Lemma 5 , the equation $\psi^{k}(x)=y_{1}$, as an equation in $x$, has solutions iff $\operatorname{Tr}_{n / 1}\left(y_{1}\right)=0$. By the assumption (11), there is at most one $y_{1}$ such that $h_{d, p}\left(y_{1}\right)=b$ and $\operatorname{Tr}_{n / 1}\left(y_{1}\right)=0$, so at most one of the equations $\psi^{k}(x)=y_{1}$ has solutions. By Lemma 5(iv), if the equation $\psi^{k}(x)=y_{1}$ has solutions then it has exactly $p$ of them, which concludes the proof that GD $f$ is $p$-to- 1 .

(ii) If $h_{d, p}$ is a permutation of $\mathbb{F}_{p^{n}}$, then in the equation (11) there is precisely one element $y$ such that $h_{d, p}(y)=b$, so the whole set has at most one element. By (i), $f$ is GAPN.

Example 2. Consider the function $f(x)=x^{13}$ over $\mathbb{F}_{3^{4}}$. We have

$$
\operatorname{GD} f(x)=-\left(x^{9}+x^{3}+x\right)=-\left(x^{3}-x\right)^{3}+\left(x^{3}-x\right)=\left(h_{13,3} \circ \psi^{2}\right)(x)
$$

with $h_{13,3}(y)=-y$. Since $h_{13,3}$ is obviously a permutation, $f$ is GAPN by Theorem 4. Alternatively, we can see that $f$ is GAPN because it has algebraic degree 3 and $d=3^{2}+3+1$, which satisfies the conditions of Theorem 1 .

Note that in this case the function $g_{13,3}$ defined by $(10)$ is $g_{13,3}=-\psi$, which is not a permutation; this shows that the converse of Theorem 3 does not hold.

Also note that the condition that $n$ is not divisible by $p$ is necessary in Theorem 4. If we consider the same function $f(x)=x^{13}$ but this time over $\mathbb{F}_{3^{3}}$, then $f$ is not GAPN. This is because $\psi^{2}(x)=x^{9}+x^{3}+x$ is not 3-to- 1 as a function over $\mathbb{F}_{3^{3}}$ (for example $\psi^{2}(\beta)=0$ for all the 9 distinct elements $\beta$ of the form $\beta=\alpha^{3}-\alpha$ with $\left.\alpha \in \mathbb{F}_{3^{3}}\right)$. 


\section{Constructions of GAPN functions as sums of GAPN monomials}

In Section 3 we considered monomial functions which are GAPN. The sum of two GAPN functions is not necessarily a GAPN function. Kuroda and Tsujie [9] and Zha et. al. [16] presented some GAPN functions which are obtained by adding monomial GAPN functions. In this section we give new constructions of this type.

Namely, we add certain Generalized Gold functions (of the type described in Section 3) of the same algebraic degree. Throughout this section we assume $p \geq 3$.

Theorem 5. Consider the field $\mathbb{F}_{p^{n}}$ and let $1 \leq u<p-1$ be such that $\operatorname{gcd}\left(u, p^{n}-\right.$ $1)=1$ and $\ell_{2}>\ell_{1}$ such that $\operatorname{gcd}\left(\ell_{2}-\ell_{1}, n\right)=1$. Consider the following GAPN monomial functions over $\mathbb{F}_{p^{n}}$, of algebraic degree $p-1+u$ :

$$
f_{i}=x^{i p^{\ell_{2}}+(p-1+u-i) p^{\ell_{1}}} \quad \text { for } u \leq i \leq p-1 .
$$

The function $f=\sum_{i=u}^{p-1} c_{i} f_{i}$ with $c_{i}$ constants in $\mathbb{F}_{p^{n}}$ is GAPN iff the following equation in a has no non-zero solutions in $\mathbb{F}_{p^{n}}$ :

$$
\sum_{i=0}^{p-1-u} c_{i+u}\left(\begin{array}{c}
p-1-u \\
i
\end{array}\right)\left(-a^{p-1}\right)^{i}=0
$$

Proof. Let $a \in \mathbb{F}_{p^{n}}, a \neq 0$. We know by Theorem 2 and Lemma 2 that

$$
\mathrm{GD}_{a} f_{i}(x)=-a^{i p^{\ell_{2}}+(p-1+u-i) p^{\ell_{1}}}\left(\begin{array}{c}
i \\
u
\end{array}\right)\left(\left(\frac{x}{a}\right)^{p^{\ell_{2}}}-\left(\frac{x}{a}\right)^{p^{\ell_{1}}}\right)^{u}
$$

and therefore

$$
\mathrm{GD}_{a} f(x)=-\left(\left(\frac{x}{a}\right)^{p^{\ell_{2}}}-\left(\frac{x}{a}\right)^{p^{\ell_{1}}}\right)^{u} \sum_{i=u}^{p-1} c_{i} a^{i p^{\ell_{2}}+(p-1+u-i) p^{\ell_{1}}}\left(\begin{array}{l}
i \\
u
\end{array}\right) .
$$

We know from the proof of Theorem 2 that $\left(x^{p^{\ell_{2}}}-x^{p^{\ell_{2}}}\right)^{u}$ is $p$-to- 1 which means that $\mathrm{GD}_{a} f(x)$ is $p$-to- 1 iff

$$
\sum_{i=u}^{p-1} c_{i} a^{i p^{\ell_{2}}+(p-1+u-i) p^{\ell_{1}}}\left(\begin{array}{l}
i \\
u
\end{array}\right) \neq 0
$$

In other words, $f$ is GAPN iff the equation in $a$

$$
\sum_{i=u}^{p-1} c_{i} a^{i p^{\ell_{2}}+(p-1+u-i) p^{\ell_{1}}}\left(\begin{array}{l}
i \\
u
\end{array}\right)=0
$$


has no non-zero solutions. We can rewrite the equation as:

$$
\begin{aligned}
& \sum_{i=u}^{p-1} c_{i} a^{i p^{\ell_{2}}+(p-1+u-i) p^{\ell_{1}}}\left(\begin{array}{l}
i \\
u
\end{array}\right)= \\
& =a^{u p^{\ell_{2}}+(p-1) p^{\ell_{1}}} \sum_{i=u}^{p-1} c_{i} a^{(i-u) p^{\ell_{2}}+(u-i) p^{\ell_{1}}}\left(\begin{array}{l}
i \\
u
\end{array}\right) \\
& =a^{u p^{\ell_{2}}+(p-1) p^{\ell_{1}}} \sum_{i=u}^{p-1} c_{i} a^{(i-u)\left(p^{\ell_{2}}-p^{\ell_{1}}\right)}\left(\begin{array}{l}
i \\
u
\end{array}\right) \\
& =0
\end{aligned}
$$

and since $a \neq 0$ this is equivalent to

$$
\sum_{i=u}^{p-1} c_{i} a^{(i-u)\left(p^{\ell_{2}}-p^{\ell_{1}}\right)}\left(\begin{array}{l}
i \\
u
\end{array}\right)=0
$$

Since $\operatorname{gcd}\left(\ell_{2}-\ell_{1}, n\right)=1$, we have that

$$
\operatorname{gcd}\left(p^{\ell_{2}}-p^{\ell_{1}}, p^{n}-1\right)=p^{\operatorname{gcd}\left(\ell_{2}-\ell_{1}, n\right)}-1=p-1 .
$$

Hence $\left(p^{\ell_{2}}-p^{\ell_{1}}\right) /(p-1)$ is invertible modulo $p^{n}-1$, and let $k$ be its inverse. Replacing $a$ by $a^{k}$ in the equation (13) we obtain

$$
\sum_{i=u}^{p-1} c_{i}\left(\begin{array}{l}
i \\
u
\end{array}\right) a^{(p-1)(i-u)}=0 .
$$

Finally, replacing the index of summation by $i-u$ and using equation (6) we have

$$
\begin{aligned}
\sum_{i=u}^{p-1} c_{i}\left(\begin{array}{l}
i \\
u
\end{array}\right) a^{(p-1)(i-u)} & =\sum_{i=0}^{p-1-u} c_{i+u}\left(\begin{array}{c}
i+u \\
u
\end{array}\right) a^{(p-1) i} \\
& =\sum_{i=0}^{p-1-u} c_{i+u}(-1)^{i+u-u}\left(\begin{array}{c}
p-1-u \\
p-1-i-u
\end{array}\right) a^{(p-1) i}
\end{aligned}
$$

which gives the equation (12) in the statement of the theorem.

We examine whether there are constants $c_{i}$ which satisfy condition (12). First we give a non-constructive proof of existence, and then we give some concrete constructions. Furthermore, for any suitable prescribed integer $s$, the next result shows the existence of a GAPN function consisting of exactly $s$ monomials.

Theorem 6. Consider the finite field $\mathbb{F}_{p^{n}}$ with $p \geq 3$ and let $1 \leq u<p-1$ be such that $\operatorname{gcd}\left(u, p^{n}-1\right)=1$. Let $2 \leq s \leq p-u$ be any prescribed integer and 
$i_{1}, i_{2}, \ldots, i_{s}$ be any choice of integers satisfying $u \leq i_{1}<i_{2}<\cdots<i_{s} \leq p-1$. Let $\ell_{2}>\ell_{1}$ such that $\operatorname{gcd}\left(\ell_{2}-\ell_{1}, n\right)=1$. Then there exits nonzero coefficients $c_{1}, \ldots, c_{s} \in \mathbb{F}_{p^{n}}^{*}$ such that the polynomial function $f: \mathbb{F}_{p^{n}} \rightarrow \mathbb{F}_{p^{n}}$ given by

$f(x)=c_{1} x^{i_{1} p^{\ell_{2}}+\left(p-1+u-i_{1}\right) p^{\ell_{1}}}+c_{2} x^{i_{2} p^{\ell_{2}}+\left(p-1+u-i_{2}\right) p^{\ell_{1}}}+\cdots+c_{s} x^{i_{s} p^{\ell_{2}}+\left(p-1+u-i_{s}\right) p^{\ell_{1}}}$, which consists of exactly s distinct monomials, is GAPN.

Proof. It follows from Theorem 5 (namely from (12)) that it is enough to prove that there are $c_{1}, \ldots, c_{s} \in \mathbb{F}_{p^{n}}^{*}$ such that the equation

$$
\begin{aligned}
& c_{1}\left(\begin{array}{c}
p-1-u \\
i_{1}-u
\end{array}\right)(-1)^{\left(i_{1}-u\right)} x^{(p-1)\left(i_{1}-u\right)}+c_{2}\left(\begin{array}{c}
p-1-u \\
i_{2}-u
\end{array}\right)(-1)^{\left(i_{2}-u\right)} x^{(p-1)\left(i_{2}-u\right)} \\
& +\cdots+c_{s}\left(\begin{array}{c}
p-1-u \\
i_{s}-u
\end{array}\right)(-1)^{\left(i_{s}-u\right)} x^{(p-1)\left(i_{s}-u\right)}=0
\end{aligned}
$$

has no nonzero solution $x \in \mathbb{F}_{p^{n}}^{*}$.

Since all the binomial coefficients in the equation above are nonzero, we can define, for convenience:

$$
\beta_{j}=(-1)^{\left(i_{j}-i_{1}\right)} \frac{c_{j}\left(\begin{array}{c}
p-1-u \\
i_{j}-u
\end{array}\right)}{c_{1}\left(\begin{array}{c}
p-1-u \\
i_{1}-u
\end{array}\right)}
$$

for $j=1, \ldots, s$. Note that $\beta_{1}=1$. For given $\beta_{2} \ldots, \beta_{s} \in \mathbb{F}_{p^{n}}^{*}$ let $\psi: \mathbb{F}_{p^{n}} \rightarrow \mathbb{F}_{p^{n}}$ be the map defined as

$$
\psi(x)=1+\beta_{2} x^{(p-1)\left(i_{2}-i_{1}\right)}+\beta_{3} x^{(p-1)\left(i_{3}-i_{1}\right)}+\cdots+\beta_{s} x^{(p-1)\left(i_{s}-i_{1}\right)} .
$$

The equation (14) has a non-zero solution $a$ iff $\psi(a)=0$. Therefore existence of $c_{1}, \ldots, c_{s} \in \mathbb{F}_{p^{n}}^{*}$ such that the equation (14) has no nonzero solutions is equivalent to the existence of $\beta_{2} \ldots, \beta_{s} \in \mathbb{F}_{p^{n}}^{*}$ such that $0 \notin \operatorname{Im} \psi$.

Let $H=\left\{x^{p-1}: x \in \mathbb{F}_{p^{n}}^{*}\right\}$ be the multiplicative subgroup of $\mathbb{F}_{p^{n}}^{*}$ of cardinality $|H|=\left(p^{n}-1\right) /(p-1)$. Note for any $a \in \mathbb{F}_{p^{n}}^{*}$ we have

$$
\psi(a)=1+\beta_{2} b^{i_{2}-i_{1}}+\cdots+\beta_{s} b^{i_{s}-i_{1}}
$$

where $b=a^{p-1} \in H$. For $b \in H$ let $R(b)$ be the subset of $\left(\mathbb{F}_{p^{n}}^{*}\right)^{s-1}$ defined as

$$
R(b)=\left\{\left(\beta_{2}, \ldots, \beta_{s}\right) \in\left(\mathbb{F}_{p^{n}}^{*}\right)^{s-1}: 1+\beta_{2} b^{i_{2}-i_{1}}+\cdots+\beta_{s} b^{i_{s}-i_{1}}=0\right\} .
$$

Note that $|R(b)| \leq\left(p^{n}-1\right)^{s-2}$. Indeed for any choice of $\beta_{2}, \ldots, \beta_{s-1} \in \mathbb{F}_{p^{n}}^{*}$ there is a unique element $\beta_{s} \in \mathbb{F}_{p^{n}}$ satisfying $1+\beta_{2} b^{i_{2}-i_{1}}+\cdots+\beta_{s} b^{i_{s}-i_{1}}=0$, which is given by

$$
\beta_{s}=-\frac{1+\beta_{2} b^{i_{2}-i_{1}}+\cdots+\beta_{s-1} b^{i_{s-1}-i_{1}}}{b^{i_{s}-i_{1}}} .
$$

Hence for any choice of $\beta_{2}, \ldots, \beta_{s-1} \in \mathbb{F}_{p^{n}}^{*}$ there is at most one $\beta_{s} \in \mathbb{F}_{p^{n}}^{*}$ such that $\left(\beta_{2}, \ldots, \beta_{s}\right) \in R(b)$. This implies that $|R(b)| \leq\left(p^{n}-1\right)^{s-2}$. 
Let $R=\cup_{b \in H} R(b)$. It is clear that

$$
|R| \leq \sum_{b \in H}|R(b)| \leq \frac{p^{n}-1}{p-1}\left(p^{n}-1\right)^{s-2}=\frac{\left(p^{n}-1\right)^{s-1}}{p-1}
$$

and therefore, since $p>2$

$$
\left|\left(\mathbb{F}_{p^{n}}^{*}\right)^{s-1} \backslash R\right| \geq\left(p^{n}-1\right)^{s-1}-\frac{\left(p^{n}-1\right)^{s-1}}{p-1}=\left(p^{n}-1\right)^{s-1} \frac{p-2}{p-1}>0
$$

For any choice of $\left(\beta_{2}, \ldots, \beta_{s}\right) \in\left(\mathbb{F}_{p^{n}}^{*}\right)^{s-1} \backslash R$ (and the $c_{i}$ chosen accordingly by putting $c_{1}$ and using (15)) we have that $\left(\beta_{2}, \ldots, \beta_{s}\right) \notin R(b)$ for any $b \in H$ and hence $\psi(a) \neq 0$ for all $a \in \mathbb{F}_{p^{n}}^{*}$. This completes the proof.

Now we give some concrete constructions.

Theorem 7. Consider the finite field $\mathbb{F}_{p^{n}}$ and let $\ell_{2}>\ell_{1}$ such that $\operatorname{gcd}\left(\ell_{2}-\right.$ $\left.\ell_{1}, n\right)=1$ and $1 \leq u<p-1$ such that $\operatorname{gcd}\left(u, p^{n}-1\right)=1$. The following function over $\mathbb{F}_{p^{n}}$ is GAPN:

$$
f(x)=\sum_{i=u}^{p-1} c^{p-1-i} x^{i p^{\ell_{2}}+(p-1+u-i) p^{\ell_{1}}}
$$

where $c=\alpha^{v}$, with $\alpha$ a primitive element in $\mathbb{F}_{p^{n}}$ and $v \mid p-1, v<p-1$ (in particular we can have $c=\alpha$ ).

Proof. Applying Theorem 5, we see that $f$ is GAPN iff the following equation in $a$ has no non-zero solution:

$$
\sum_{i=0}^{p-1-u} c^{p-1-u-i}\left(\begin{array}{c}
p-1-u \\
i
\end{array}\right)\left(-a^{p-1}\right)^{i}=0
$$

This is equivalent to

$$
\left(c-a^{p-1}\right)^{p-1-u}=0
$$

which in turn has a solution $a$ iff

$$
a^{p-1}=c
$$

However, note that $c$ was chosen so that it has order exactly $\frac{p^{n}-1}{v}$ whereas the order of $a^{p-1}$ is a factor of $\frac{p^{n}-1}{p-1}$, which is strictly smaller than the order of $c$. So the equation has no solutions.

Next we consider the particular case of Theorem 5 where we sum exactly two functions. Here we completely classify GAPN functions in the corresponding class by an iff condition. 
Theorem 8. Consider the finite field $\mathbb{F}_{p^{n}}$ and let $c \in \mathbb{F}_{p^{n}}^{*}$. Let $\ell_{2}>\ell_{1}$ such that $\operatorname{gcd}\left(\ell_{2}-\ell_{1}, n\right)=1$ and $1 \leq u \leq i_{1}<i_{2} \leq p-1$ such that $\operatorname{gcd}\left(u, p^{n}-1\right)=1$. The following function over $\mathbb{F}_{p^{n}}$

$$
f(x)=c x^{i_{1} p^{\ell_{2}}+\left(p-1+u-i_{1}\right) p^{\ell_{1}}}+x^{i_{2} p^{\ell_{2}}+\left(p-1+u-i_{2}\right) p^{\ell_{1}}}
$$

is GAPN iff

$$
c=(-1)^{i_{2}-i_{1}+1} \frac{\left(\begin{array}{c}
p-1-u \\
i_{2}-u
\end{array}\right)}{\left(\begin{array}{c}
p-1-u \\
i_{1}-u
\end{array}\right)} \beta
$$

for some element $\beta \in \mathbb{F}_{p^{n}}$ such that the order of $\beta$ does not divide $\frac{p^{n}-1}{i_{3}(p-1)}$, where $i_{3}=\operatorname{gcd}\left(i_{2}-i_{1}, \frac{p^{n}-1}{p-1}\right)$ (in other words, $\beta \notin\left\langle\alpha^{(p-1) i_{3}}\right\rangle$, where and $\alpha$ is a primitive element of $\left.\mathbb{F}_{p^{n}}\right)$.

Proof. Using Theorem 5, $f$ is GAPN iff the following equation has no non-zero solutions:

$$
c\left(\begin{array}{c}
p-1-u \\
i_{1}-u
\end{array}\right)\left(-a^{p-1}\right)^{i_{1}-u}+\left(\begin{array}{c}
p-1-u \\
i_{2}-u
\end{array}\right)\left(-a^{p-1}\right)^{i_{2}-u}=0 .
$$

Assume, for a contradiction, that there was a nonzero solution $a$. Dividing by $\left(-a^{p-1}\right)^{i_{1}-u}$ we obtain

$$
\left(a^{p-1}\right)^{i_{2}-i_{1}}=(-1)^{i_{2}-i_{1}+1} c\left(\begin{array}{c}
p-1-u \\
i_{1}-u
\end{array}\right)\left(\begin{array}{c}
p-1-u \\
i_{2}-u
\end{array}\right)^{-1}=\beta
$$

Note that for any non-zero $a \in \mathbb{F}_{p^{n}}$ we have $a^{p-1} \in\left\langle\alpha^{p-1}\right\rangle$, and therefore $\left(a^{p-1}\right)^{i_{2}-i_{1}} \in\left\langle\alpha^{(p-1) i_{3}}\right\rangle$. Contradiction.

Corollary 1. With the notations from Theorem 8, a sufficient condition for $f$ to be GAPN is that $\operatorname{gcd}\left(i_{2}-i_{1}, \frac{p^{n}-1}{p-1}\right)=1, n$ is not a multiple of $p-1$ and

$$
c=(-1)^{i_{2}-i_{1}+1} \frac{\left(\begin{array}{c}
p-1-u \\
i_{2}-u
\end{array}\right)}{\left(\begin{array}{c}
p-1-u \\
i_{1}-u
\end{array}\right)} \beta
$$

where $\beta \neq 0$ is chosen from the base field $\mathbb{F}_{p}$ so that its order does not divide $\operatorname{gcd}(n, p-1)$ (for example $\beta$ is primitive in $\mathbb{F}_{p}$ ).

Proof. If $\beta \in \mathbb{F}_{p}^{*}$ then $c \in \mathbb{F}_{p}^{*}$. We compute

$$
\begin{aligned}
\left\langle\alpha^{(p-1)}\right\rangle \cap \mathbb{F}_{p}^{*} & =\left\{x \in \mathbb{F}_{p^{n}}^{*}: \operatorname{ord}(x) \mid \frac{p^{n}-1}{p-1} \text { and } \operatorname{ord}(x) \mid(p-1)\right\} \\
& =\left\{x \in \mathbb{F}_{p^{n}}^{*}: \operatorname{ord}(x) \mid \operatorname{gcd}\left(p-1, p^{n-1}+p^{n-2}+\ldots+1\right)\right\} \\
& =\left\{x \in \mathbb{F}_{p}^{*}: \operatorname{ord}(x) \mid \operatorname{gcd}(p-1, n)\right\}
\end{aligned}
$$


Remark 2. Corollary 1 above includes, as a particular case, [16, Proposition 2.7]. Namely, in their result the following assumptions are made (using our notations above): $i_{2}-i_{1}=1, c=1, \operatorname{gcd}(n, p-1)$ equals 1 or 2 , and $2 i_{1}-u+2 \neq p$.

Using $i_{2}=i_{1}+1$ and $c=1$ we have $\beta=\left(\begin{array}{c}p-1-u \\ i_{1}-u\end{array}\right)\left(\begin{array}{c}p-1-u \\ i_{2}-u\end{array}\right)^{-1}=\frac{i_{1}-u+1}{p-1-i_{1}}$. Corollary 1 states that a sufficient condition for $f$ being GAPN is that the order of $\beta$ does not divide $\operatorname{gcd}(n, p-1)$, which in this case was assumed to be 1 or 2 . This is equivalent to $\beta$ being different from 1 and from -1 . We have

$$
\frac{i_{1}-u+1}{p-1-i_{1}}=1 \Leftrightarrow 2 i_{2}-u-2=p
$$

and

$$
\frac{i_{1}-u+1}{p-1-i_{1}}=-1 \Leftrightarrow u=p,
$$

neither of which are possible, so $f$ is indeed GAPN.

\section{New GAPN functions of degree $p$ with many terms and some characterizations}

In Theorem 1 we characterized monomial GAPN functions of degree $p$. In this section we characterize some GAPN functions of degree $p$ with many terms and give some new examples. In particular we obtain new GAPN functions of degree $p$ having $(s+1)(s+2) / 2$ distinct terms for each $2 \leq s \leq p-1$.

Our candidates for GAPN functions are of the form $f(x)=x^{p-s} L(x)^{s}$ with $1 \leq s \leq p-1$ and $L(x)$ being a linearized polynomial. Note that this case covers all functions of the form $g(x)=M(x)^{p-s} N(x)^{s}$ with $M, N$ being linearized polynomials and at least one of them, say $M(x)$, being invertible; $g$ is EAequivalent to $\left(g \circ M^{-1}\right)(x)=x^{p-s}\left(\left(N \circ M^{-1}\right)(x)\right)^{s}$ and $N \circ M^{-1}$ is linearized.

For $p=2$, APN functions of this form have been characterized in [1], and it was shown that, essentially, no new functions can be obtained by this method beyond the monomial APN functions. The results in this section extend this result to $p>2$ and explain some differences compared to the $p=2$ case.

In order to present our results in a general perspective we assume that $p$ is an arbitrary prime (possibly $p=2$ as well) in this section. Let $L(x)=a_{0} x+$ $a_{1} x^{p}+\cdots+a_{n-1} x^{p^{n-1}} \in \mathbb{F}_{p^{n}}[x]$ be an arbitrary linearized polynomial. Let $L_{1}(x) \in \mathbb{F}_{p^{n}}[x]$ be the linearized polynomial

$$
L_{1}(x)=a_{1} x^{p}+\cdots+a_{n-1} x^{p^{n-1}} \in \mathbb{F}_{p^{n}}[x]
$$

obtained from $L(x)$ by removing the first term $a_{0} x$.

For $p=2$, a basic candidate for a quadratic APN is of the form

$$
f(x)=x L(x) .
$$

Since $f(x)=x L_{1}(x)+a_{0} x^{2}$ and $a_{0} x^{2}$ is affine, $f$ is EA-equivalent to

$$
f_{1}(x)=x L_{1}(x) .
$$


The important result $[1$, Theorem 6$]$ on quadratic APN functions states that $f_{1}$ is an APN function iff

$$
L_{1}(x)=\alpha x^{2^{k}} \text { with } \alpha \in \mathbb{F}_{2^{n}}^{*} \text { and } \operatorname{gcd}(k, n)=1 .
$$

This implies that if $f_{1}$ is an APN function, then

$$
L_{1} \text { is a permutation on } \mathbb{F}_{2^{n}} .
$$

Let $\psi, \psi_{1}: \mathbb{F}_{p^{n}}^{*} \rightarrow \mathbb{F}_{p^{n}}$ be the maps given by

$$
\psi(x)=\frac{L(x)}{x} \text { and } \psi_{1}(x)=\frac{L_{1}(x)}{x} .
$$

We have

$$
\psi(x)=\psi_{1}(x)+a_{0} .
$$

We call that $\psi$ (respectively $\psi_{1}$ ) is $(p-1)$-to- 1 if the preimage $\psi^{-1}(a)$ (resp. $\left.\psi_{1}^{-1}(a)\right)$ has cardinality either 0 or $p-1$ for all $a \in \mathbb{F}_{p^{n}}$. It follows from (20) that $\psi$ is $(p-1)$-to- 1 iff $\psi_{1}$ is $(p-1)$-to- 1 .

We start with a useful lemma. We denote the evaluation maps $x \rightarrow L(x)$ and $x \rightarrow L_{1}(x)$ on $\mathbb{F}_{p^{n}}$ by $L$ and $L_{1}$ as well as the corresponding linearized polynomials.

Lemma 6. Under notation as above assume that $\psi_{1}$ is $(p-1)$-to-1. Then we have the following:

i) If $p=2$, then $L_{1}$ is a permutation map or equivalently $\operatorname{dim}_{\mathbb{F}_{2}} \operatorname{Ker} L_{1}=0$.

ii) If $p>2$, then $\operatorname{dim}_{\mathbb{F}_{p}}$ Ker $L_{1} \in\{0,1\}$.

iii) If $p>2$, then $\operatorname{dim}_{\mathbb{F}_{p}}$ Ker $L \in\{0,1\}$.

Proof. We first prove items i) and ii). If $\operatorname{dim}_{\mathbb{F}_{p}}$ Ker $L_{1} \geq 2$, then the preimage $\psi_{1}^{-1}(0)$ has at least $p^{2}-1>p-1$ elements, which is a contradiction. The result in item i) follows from [1, Lemma 1]. Finally we prove item iii). As $\psi_{1}$ is $(p-1)$ to- 1 , it follows from $(20)$ that $\psi$ is $(p-1)$-to- 1 as well. As in the argument in the proof of item ii), if $\operatorname{dim}_{\mathbb{F}_{p}} \operatorname{Ker} L \geq 2$, then the preimage $\psi^{-1}(0)$ has at least $p^{2}-1>p-1$ elements, which is a contradiction. This completes the proof.

When we consider the function $f(x)=x^{p-s} L(x)^{s}$ for $p>2$ there are some differences between the case $s=1$ and $s \geq 2$, so we shall consider them separately. For $s=1$, note firstly that $f$ is EA-equivalent to $f_{1}(x)=x^{p-1} L_{1}(x)$ as $f(x)=x^{p-1} L_{1}(x)+a_{0} x^{p}$ and $a_{0} x^{p}$ is affine. Therefore $f$ is a GAPN function iff $f_{1}$ is a GAPN function. Next we present our first extension of the result in (18) on quadratic APN functions.

Theorem 9. Let $L_{1}(x) \in \mathbb{F}_{p^{n}}[x]$ be a linearized polynomial of the form $L_{1}(x)=$ $\sum_{\mathbb{F}}^{n-1} a_{i} x^{p^{i}}$ (i.e. the monomial $x$ is missing). Let $f_{1}: \mathbb{F}_{p^{n}} \rightarrow \mathbb{F}_{p^{n}}$ and $\psi_{1}: \mathbb{F}_{p^{n}}^{*} \rightarrow$ $\mathbb{F}_{p^{n}}$ be the maps given by

$$
f_{1}(x)=x^{p-1} L_{1}(x) \text { and } \psi_{1}(x)=\frac{L_{1}(x)}{x} .
$$

Then $f_{1}$ is a GAPN function iff $\psi_{1}$ is $(p-1)$-to-1. 
Remark 3. In Example 6 below we construct $L_{1}(x)$ in the form (17) such that $f_{1}$ is a GAPN function and $\operatorname{dim}_{\mathbb{F}_{p}}$ Ker $L_{1}=1$, i.e. $L_{1}$ is not a permutation on $\mathbb{F}_{p^{n}}$. This is a difference to the the quadratic APN results in (18) and (19).

Remark 4. In Example 6 below we construct $L_{1}(x)$ in the form (17) such that $f_{1}$ is a GAPN function and $L_{1}$ is not monomial (both for $\operatorname{dim}_{\mathbb{F}_{p}} \operatorname{Ker} L_{1}=0$ and 1). This is another difference to the the quadratic APN result in (18).

Remark 5. An important result of Hou [5] implies the following: Let $L_{1}(x) \in$ $\mathbb{F}_{p^{n}}[x]$ be a linearized polynomial of the form (17). Assume that $L_{1}$ is a permutation on $\mathbb{F}_{p^{n}}$. Then the map

$$
[x] \in \mathbb{F}_{p^{n}}^{*} / \mathbb{F}_{p}^{*} \rightarrow\left[L_{1}(x) / x\right] \in \mathbb{F}_{p^{n}}^{*} / \mathbb{F}_{p}^{*}
$$

is a permutation iff $L_{1}(x)=\alpha x^{p^{k}}$ with $\alpha \in \mathbb{F}_{p^{n}}^{*}$ and $\operatorname{gcd}(k(p-1), n)=1$. Here $[x]$ denotes the equivalence class of the quotient multiplicative group $\mathbb{F}_{p^{*}}^{*} / \mathbb{F}_{p}^{*}$ and it is easy to observe that the map in (21) is well defined.

If $p=2$, then the map in (21) is a permutation iff $\psi_{1}$ is 1 -to-1. Moreover it follows from Lemma 6 , item i) that the extra assumption that $L_{1}$ is a permutation is not necessary, as it holds automatically when $\psi_{1}$ is 1-to- 1 .

However when $p>2$, the condition that the map in (21) is a permutation is strictly stronger than the condition that $\psi_{1}$ is $(p-1)$-to- 1 . For instance in Examples 3, 4, 6 below we obtain many functions $\psi_{1}$ which are $(p-1)$-to- 1 but $L_{1}$ is not a monomial. Even among the monomial functions, when $\operatorname{gcd}(k, n)=1$ and $\operatorname{gcd}(p-1, n)>1$, let $L_{1}(x)=\alpha x^{p^{k}}$ with $\alpha \in \mathbb{F}_{p^{n}}^{*}$. Then the map in (21) is not a permutation while $\psi_{1}$ is $(p-1)$-to- 1 .

These arguments explain some interesting differences in the situations of $p=2$ and $p>2$. Moreover we obtain that Theorem 9 coincides exactly with the result in (18) on quadratic APN functions. Hence Theorem 9 is a generalization of the the result in (18) on quadratic APN functions.

We prove Theorem 9 after the proof of the next theorem, which deals with the case $s \geq 2$ and is our second extension of the result in (18) on quadratic APN functions. Note that in the case $s \geq 2$ we cannot, in general, replace $L(x)$ by $L_{1}(x)$, as $x^{p-s} L(x)^{s}$ is not necessarily EA-equivalent to $x^{p-s} L_{1}(x)^{s}$.

Theorem 10. Let $p$ be an odd prime. Let $2 \leq s \leq p-1$ be an integer. Let $L(x) \in \mathbb{F}_{p^{n}}[x]$ be an arbitrary linearized polynomial. Let $f: \mathbb{F}_{p^{n}} \rightarrow \mathbb{F}_{p^{n}}$ and $\psi: \mathbb{F}_{p^{n}} \rightarrow \mathbb{F}_{p^{n}}$ be the maps given by

$$
f(x)=x^{p-s} L(x)^{s} \text { and } \psi(x)=\frac{L(x)}{x} .
$$

Then $f$ is a GAPN function iff $L$ is a permutation on $\mathbb{F}_{p^{n}}$ and $\psi$ is $(p-1)$-to-1.

Remark 6. In Examples 3, 4, 5 below, using Theorem 10 we obtain new degree $p$ GAPN functions with many distinct terms. 
We use the following two lemmas in the proofs of Theorems 9 and 10 .

Lemma 7. Let $L(x) \in \mathbb{F}_{p^{n}}[x]$ be a linearized polynomial, which is not the zero polynomial. Let $k$ be an integer, $1 \leq k \leq p-1$ and let $a \in \mathbb{F}_{p^{n}}$. We have

$$
\mathrm{D}_{a}^{(k)}\left(L(x)^{k}\right)=k ! L(a)^{k} .
$$

Moreover if $k \geq 2$ we also have

$$
\mathrm{D}_{a}^{(k-1)}\left(L(x)^{k}\right)=k ! L(a)^{k-1} L(x)+c \text {, where } c \in \mathbb{F}_{p^{n}} .
$$

Proof. For integers $1 \leq m \leq k-1$ we have

$$
\mathrm{D}_{a}^{(m)}\left(L(x)^{k}\right)=k(k-1) \cdots(k-m+1) L(a)^{m} L(x)^{k-m}+\operatorname{lot},
$$

where lot stands for lower order terms, in the sense of lower algebraic degree. We prove (24) by induction on $m$. Putting $m=k-1$ we obtain (23). Taking the derivative of (23) we get (22)

Lemma 8. Let $g_{1}, g_{2}: \mathbb{F}_{p^{n}} \rightarrow \mathbb{F}_{p^{n}}$ be arbitrary functions. Let $k$ be a positive integer. Let $a \in \mathbb{F}_{p^{n}}^{*}$. We have

$$
\mathrm{D}_{a}^{(k)}\left(g_{1}(x) g_{2}(x)\right)=\sum_{j=0}^{k}\left(\begin{array}{l}
k \\
j
\end{array}\right) \mathrm{D}_{a}^{(j)}\left(g_{1}(x)\right) \mathrm{D}_{a}^{(k-j)}\left(g_{2}(x+j a)\right) .
$$

Proof. The proof follows from a rather straightforward induction on $k$.

Remark 7. As $\mathrm{D}_{a}^{(k)}\left(g_{1}(x) g_{2}(x)\right)=\mathrm{D}_{a}^{(k)}\left(g_{2}(x) g_{1}(x)\right)$, the right hand side of $(25)$ is invariant under the transformation $g_{1} \leftrightarrow g_{2}$.

We first prove Theorem 10.

Proof (of Theorem 10). Assume that $L$ is not the zero polynomial. Let $a \in \mathbb{F}_{p^{n}}^{*}$. Recall that $\mathrm{GD}_{a} f(x)=\mathrm{D}_{a}^{(p-1)}(f(x))$. Using Lemma 8 and the fact that $\left(\begin{array}{c}p-1 \\ j\end{array}\right)=$ $(-1)^{j}$ in fields of characteristic $p$, we have

$$
\mathrm{GD}_{a} f(x)=\sum_{j=0}^{p-1}(-1)^{j} \mathrm{D}_{a}^{(j)}\left(x^{p-s}\right) \mathrm{D}_{a}^{(p-1-j)}\left(L(x+j a)^{s}\right) .
$$

Note that $\mathrm{D}_{a}^{(j)}\left(x^{p-s}\right)=0$ if $j>p-s$. Similarly $\mathrm{D}_{a}^{(p-1-j)}\left(L(x+j a)^{s}\right)=0$ if $p-1-j>s$. Hence the summation in the right hand side of (26) has only the terms $j=p-s-1$ and $j=p-s$ :

$$
\begin{aligned}
\mathrm{GD}_{a} f(x)= & (-1)^{p-s-1} \mathrm{D}_{a}^{(p-s-1)}\left(x^{p-s}\right) \mathrm{D}_{a}^{(s)}\left(L(x+(p-s-1) a)^{s}\right) \\
& +(-1)^{p-s} \mathrm{D}_{a}^{(p-s)}\left(x^{p-s}\right) \mathrm{D}_{a}^{(s-1)}\left(L(x+(p-s) a)^{s}\right) .
\end{aligned}
$$


Using Lemma 7 we obtain that for some constants $c, c_{1}, c_{2} \in \mathbb{F}_{p^{n}}$ :

$$
\begin{aligned}
\mathrm{GD}_{a} f(x)= & (-1)^{s}\left((p-s) ! a^{p-s-1} x+c_{1}\right)\left(s ! L(a)^{s}\right) \\
& +(-1)^{s-1}(p-s) ! a^{p-s}\left(s ! L(a)^{s-1} L(x)+c_{2}\right) \\
= & (-1)^{s}(p-s) ! s ! a^{p-s-1} L(a)^{s-1}(x L(a)-a L(x))+c .
\end{aligned}
$$

Assume that $L$ is not a permutation, i.e. Ker $L \neq\{0\}$ and choose $a \in \mathbb{F}_{p^{n}}^{*}$ such that $L(a)=0$. Using $(28)$ we obtain that $\mathrm{GD}_{a} f(x)=c$ and hence $f$ cannot be a GAPN function. Assume next that $L$ is a permutation. From (28), taking into account that $(p-s) ! s ! a^{p-s-1} L(a)^{s-1} \neq 0$ we conclude that $f$ is a GAPN function iff the equation

$$
x L(a)-a L(x)=0
$$

has at most $p$ solutions $x \in \mathbb{F}_{p^{n}}$ for each $a \in \mathbb{F}_{p^{n}}^{*}$. This means exactly that $\psi$ is $(p-1)$-to- 1 .

Next we prove Theorem 9 .

Proof (of Theorem 9). We follow the same arguments as in the proof of Theorem 10, using $L_{1}$ instead of $L$ and taking into account that now $s=1$. Equation (28) becomes

$$
\mathrm{GD}_{a} f(x)=a^{p-2}\left(x L_{1}(a)-a L_{1}(x)\right)+c .
$$

Since $a^{p-2} \neq 0$ we conclude that $f_{1}$ is a GAPN function iff the equation

$$
x L_{1}(a)-a L_{1}(x)=0
$$

has at most $p$ solutions $x \in \mathbb{F}_{p^{n}}$ for each $a \in \mathbb{F}_{p^{n}}^{*}$, i.e. $\psi_{1}$ is $(p-1)$-to- 1 . In particular it is not necessary that $L_{1}$ is a permutation. This completes the proof.

Theorem 10 requires a linearized function which is a permutation on $\mathbb{F}_{p^{n}}$. Obviously, not all linearized functions are permutations, but, interestingly, for $p>2$, we can make them become a permutation by simply "adjusting" the coefficient of the term $x$ :

Proposition 3. Let $p$ be a prime, $p>2$, and $M(x)$ be a linearized function. There is a constant $b \in \mathbb{F}_{p^{n}}^{*}$ such that $L(x)=M(x)+b x$ is a permutation on $\mathbb{F}_{p^{n}}$. More precisely, the set $\mathbb{F}_{p^{n}}^{*} \backslash\left\{-M(\mu) / \mu: \mu \in \mathbb{F}_{p^{n}}^{*}\right\}$ has at least $\left(p^{n}-1\right) \frac{p-2}{p-1}>0$ elements, and for each of those elements $b$ the function $L(x)=M(x)+b x$ is a permutation on $\mathbb{F}_{p^{n}}$.

Proof. Choose $b$ from the set in the statement and assume, for a contradiction, that $L$ is not a permutation. Since $L$ is linearized, this means that $\operatorname{Ker} L \neq\{0\}$. Let $\mu \in \operatorname{Ker} L, \mu \neq 0$. Hence $L(\mu)=M(\mu)+b \mu=\mu(M(\mu) / \mu+b)=0$ and therefore $b=-M(\mu) / \mu$, contradicting the choice of $b$. 
It remains to prove the statement regarding the cardinality of the set. Writing $M(x)=\sum_{i=0}^{n-1} b_{i} x^{p^{i}}$ we have:

$$
\frac{M(\mu)}{\mu}=\sum_{i=0}^{n-1} b_{i} \mu^{p^{i}-1}=\sum_{i=0}^{n-1} b_{i} \alpha^{\frac{p^{i}-1}{p-1}}
$$

where $\alpha=\mu^{p-1}$. Note that $\frac{M(\mu)}{\mu}$ only depends on the value of $\alpha=\mu^{p-1}$. Since $\alpha \in\left\{\mu^{p-1}: \mu \in \mathbb{F}_{p^{n}}^{*}\right\}, \alpha$ will take exactly $\left(p^{n}-1\right) /(p-1)$ values, and therefore the set $\left\{-M(\mu) / \mu: \mu \in \mathbb{F}_{p^{n}}^{*}\right\}$ has at most $\left(p^{n}-1\right) /(p-1)$ values. This concludes the proof.

In Proposition 3 above, note that $M(x) / x$ is $(p-1)$-to- 1 iff $L(x) / x$ is $(p-1)$-to- 1 , see $(20)$. Combined with Theorem 10 we obtain therefore the following general method for constructing GAPN functions:

Corollary 2. Let $L_{1}(x)=\sum_{i=1}^{n-1} a_{i} x^{p^{i}}$. If $\psi_{1}(x)=L_{1}(x) / x$ is $(p-1)$-to-1 then for all $b \in \mathbb{F}_{p^{n}} \backslash\left\{-\psi_{1}(\mu): \mu \in \mathbb{F}_{p^{n}}^{*}\right\}$ and for all $2 \leq s \leq p-1$ we have that $f(x)=x^{p-s}\left(L_{1}(x)+b x\right)^{s}$ is a GAPN function.

We need therefore to find functions $L_{1}$ such that $\psi_{1}$ is $(p-1)$-to- 1 . A large class of such functions is described in the following:

Theorem 11. Let $p$ be an odd prime. Let $n \geq 3$ and $1 \leq k \leq n-1$ be integers such that $\operatorname{gcd}(k, n)=1$. For $a \in \mathbb{F}_{p^{n}}^{*}$ let $L_{1}(x)=a x^{p^{k}}+x^{p^{n-k}}$ and $\psi_{1}: \mathbb{F}_{p^{n}}^{*} \rightarrow$ $\mathbb{F}_{p^{n}}$ given by $\psi_{1}(x)=L_{1}(x) / x$. Let $H=\left\{x^{p-1}: x \in \mathbb{F}_{p^{n}}^{*}\right\}$ be the multiplicative subgroup of $\mathbb{F}_{p^{n}}^{*}$ with $|H|=\left(p^{n}-1\right) /(p-1)$. Then we have the following:

i) If $a^{\left(p^{n}-1\right) /(p-1)} \neq 1$ (i.e. $\left.a \notin H\right)$ then $\psi_{1}$ is $(p-1)$-to-1.

ii) If $n$ is odd and $a^{\left(p^{n}-1\right) /(p-1)}=1$ (i.e. $\left.a \in H\right)$, then $\psi_{1}$ is not $(p-1)-$ to- 1 .

iii) If $n$ is odd, then $\operatorname{dim}_{\mathbb{F}_{p}} \operatorname{Ker} L_{1}=\left\{\begin{array}{lr}0 \text { if } a^{\left(p^{n}-1\right) /(p-1)} \neq-1, \\ 1 & \text { otherwise. }\end{array}\right.$

iv) If $n$ is even, then $\operatorname{dim}_{\mathbb{F}_{p}} \operatorname{Ker} L_{1}=\left\{\begin{array}{l}0 \text { if }(-a)^{\left(p^{n}-1\right) /\left(p^{2}-1\right)} \neq 1, \\ 2 \quad \text { otherwise. }\end{array}\right.$

Proof. Note that $L_{1}(x)=0$ has a solution $x \in \mathbb{F}_{p^{n}}^{*}$ iff

$$
-a=x^{p^{n-k}-p^{k}}
$$

We have

$$
\operatorname{gcd}\left(p^{n-k}-p^{k}, p^{n}-1\right)=\left\{\begin{array}{l}
p-1 \text { if } n \text { is odd } \\
p^{2}-1 \text { if } n \text { is even. }
\end{array}\right.
$$

Combining (29) and (30) we complete the proofs of the items iii) and iv). Let $\eta_{1}: \mathbb{F}_{p^{n}}^{*} \rightarrow H$ and $\eta_{2}: H \rightarrow \mathbb{F}_{p^{n}}$ be the maps given by

$$
\eta_{2}(x)=a x+\frac{1}{x^{p^{n-k}}} \quad \text { and } \quad \eta_{1}(x)=x^{p^{k}-1} .
$$


It is easy to observe that $\eta_{1}$ is well defined and surjective. Moreover $\psi_{1}(x)=$ $\eta_{2} \circ \eta_{1}(x)$. Hence $\psi_{1}$ is $(p-1)$-to- 1 iff $\eta_{2}$ is injective.

For $x, y \in H$ we have

$$
\eta_{2}(x)=\eta_{2}(y) \Longleftrightarrow a(x-y)=\frac{(x-y)^{p^{n-k}}}{x^{p^{n-k}} y^{p^{n-k}}} .
$$

If $x \neq y$, then using (31) we get

$$
a^{\frac{p^{n}-1}{p-1}}=\left(\frac{(x-y)^{p^{n-k}-1}}{x^{p^{n-k}} y^{p^{n-k}}}\right)^{\frac{p^{n}-1}{p-1}}=1,
$$

which is a contradiction to the condition in item i). This completes the proof of item i).

Finally assume that $a \in H$. Choose $\theta \in H \backslash\{1\}$ and consider the equation

$$
a=\frac{(1-\theta)^{p^{n-k}-1}}{\theta^{p^{n-k}}} \frac{1}{x^{p^{n-k}+1}} .
$$

Note that $a,(1-\theta)^{p^{n-k}-1}, \theta \in H$. Moreover $\operatorname{gcd}\left(p^{n-k}+1,|H|\right)=1$ as $n$ is odd. Therefore there exists $x \in H$ satisfying (32). Put $y=\theta x \in H \backslash\{x\}$. Using (31) and (32) we conclude that $\eta_{2}(x)=\eta_{2}(y)$. Hence $\psi_{1}$ is not $(p-1)$-to- 1 . This completes the proof.

Remark 8. If $n$ is odd and $a=-1$, then Theorem 11 item i) corresponds to [9, Lemma 3.6].

As an application of Corollary 2 and Theorem 11 we present our first class of new GAPN functions of degree $p$.

Example 3. Let $p$ be an odd prime. Let $n \geq 3$ and $1 \leq k \leq n-1$ be integers such that $\operatorname{gcd}(k, n)=1$. Let $H$ be the multiplicative subgroup of $\mathbb{F}_{p^{*}}^{*}$ with $|H|=\left(p^{n}-1\right) /(p-1)$. Let $a \in \mathbb{F}_{p^{n}}^{*} \backslash H$ and $a_{0} \in \mathbb{F}_{p^{n}}^{*} \backslash\left\{-L_{1}(\mu) / \mu: \mu \in \mathbb{F}_{p^{n}}^{*}\right\}$ where $L_{1}(x)=a x^{p^{k}}+x^{p^{n-k}}$. For $2 \leq s \leq p-1$ let $f: \mathbb{F}_{p^{n}} \rightarrow \mathbb{F}_{p^{n}}$ be the map given by

$$
f(x)=x^{p-s}\left(a x^{p^{k}}+x^{p^{n-k}}+a_{0} x\right)^{s} .
$$

Then $f$ is a GAPN function of degree $p$ having exactly $(s+2)(s+1) / 2$ distinct terms. In particular for $s=p-1$ we have that $f$ is a GAPN function of degree $p$ having exactly $(p+1) p / 2$ distinct terms. Moreover, for each $s$, the number of functions in this class is at least

$$
\left(p^{n}-1-\frac{p^{n}-1}{p-1}\right)^{2}=\left(p^{n}-1\right)^{2} \frac{(p-2)^{2}}{(p-1)^{2}} .
$$

Indeed the number of choices of $a \in \mathbb{F}_{p^{n}}^{*} \backslash H$ is $p^{n}-1-\frac{p^{n}-1}{p-1}$. For each such $a$, there exist at least $p^{n}-1-\frac{p^{n}-1}{p-1}$ choices for $a_{0}$ (see Proposition 3 ). Therefore the number of distinct polynomials

$$
L(x)=a x^{p^{k}}+x^{p^{n-k}}+a_{0} x
$$


with $a$ and $a_{0}$ chosen as above is at least the number in (33). Let $L(x)=$ $a x^{p^{k}}+x^{p^{n-k}}+a_{0} x$ and $\tilde{L}(x)=b x^{p^{k}}+x^{p^{n-k}}+b_{0} x$ be any two choices of such polynomials. Let $f(x)=x^{p-s} L(x)^{s}$ and $\tilde{f}(x)=x^{p-s} \tilde{L}(x)^{s}$. In order to prove that, for each $s$, the number of functions in this class is at least the number in (33), it is enough to show that

$$
f=\tilde{f} \text { as maps on } \mathbb{F}_{p^{n}} \Rightarrow a=b \text { and } a_{0}=b_{0} .
$$

Assume that $f=\tilde{f}$ as maps on $\mathbb{F}_{p^{n}}$. Let $\alpha \in \mathbb{F}_{p^{n}}^{*}$. Then we have

$$
\mathrm{GD}_{\alpha} f(x)=\mathrm{GD}_{\alpha} \tilde{f}(x) \text { for all } x \in \mathbb{F}_{p^{n}}
$$

and $f(\alpha)=\alpha^{p-s} L(\alpha)^{s}=\alpha^{p-s} \tilde{L}(\alpha)^{s}$. In particular we also have

$$
L(\alpha)^{s}=\tilde{L}(\alpha)^{s} .
$$

Using the proof of Theorem 10, in particular (28), we obtain there exist functions $c, \tilde{c}: \mathbb{F}_{p^{n}}^{*} \rightarrow \mathbb{F}_{p^{n}}$ such that

$$
\mathrm{GD}_{\alpha} f(x)=(-1)^{s-1}(p-s) ! \alpha^{p-s-1} L(\alpha)^{s-1}[-x L(\alpha)+\alpha L(x)]+c(\alpha)
$$

and

$$
\mathrm{GD}_{\alpha} \tilde{f}(x)=(-1)^{s-1}(p-s) ! \alpha^{p-s-1} \tilde{L}(\alpha)^{s-1}[-x \tilde{L}(\alpha)+\alpha \tilde{L}(x)]+\tilde{c}(\alpha)
$$

for all $\alpha \in \mathbb{F}_{p^{n}}^{*}$ and $x \in \mathbb{F}_{p^{n}}$. Note that $L(0)=\tilde{L}(0)=0$ and $L(\alpha) \neq 0$, $\tilde{L}(\alpha) \neq 0$ for $\alpha \in \mathbb{F}_{p^{n}}^{*}$ as $L$ and $\tilde{L}$ are permutations on $\mathbb{F}_{p^{n}}$ by Proposition 3. Put $A(\alpha)=(-1)^{s-1}(p-s) ! \alpha^{p-s-1} L(\alpha)^{s}$ and $\tilde{A}(\alpha)=(-1)^{s-1}(p-s) ! \alpha^{p-s-1} \tilde{L}(\alpha)^{s}$ for $\alpha \in \mathbb{F}_{p^{n}}^{*}$. Using (36) and the arguments above we get $A(\alpha)=\tilde{A}(\alpha) \neq 0$ for all $\alpha \in \mathbb{F}_{p^{n}}$. Dividing (37) by $A(\alpha)$, dividing (38) by $\tilde{A}(\alpha)$, and using (35) we conclude that

$$
-x+\frac{\alpha}{L(\alpha)} L(x)+\frac{c(\alpha)}{A(\alpha)}=-x+\frac{\alpha}{\tilde{L}(\alpha)} L(x)+\frac{\tilde{c}(\alpha)}{\tilde{A}(\alpha)}
$$

for all $\alpha \in \mathbb{F}_{p^{n}}^{*}$ and $x \in \mathbb{F}_{p^{n}}$. Using (39) and the definitions of $L(x)$ and $\tilde{L}(x)$ we obtain that

$$
\begin{aligned}
& -x+\frac{\alpha}{L(\alpha)} a x^{p^{k}}+\frac{\alpha}{L(\alpha)} x^{p^{n-k}}+\frac{\alpha}{L(\alpha)} a_{0} x+\frac{c(\alpha)}{A(\alpha)} \\
& =-x+\frac{\alpha}{\tilde{L}(\alpha)} b x^{p^{k}}+\frac{\alpha}{\tilde{L}(\alpha)} x^{p^{n-k}}+\frac{\alpha}{\tilde{L}(\alpha)} b_{0} x+\frac{\tilde{c}(\alpha)}{\tilde{A}(\alpha)}
\end{aligned}
$$

for all $\alpha \in \mathbb{F}_{p^{n}}^{*}$ and $x \in \mathbb{F}_{p^{n}}$. For each $\alpha \in \mathbb{F}_{p^{n}}^{*}$, next we consider both sides of (40) as univariate polynomials in $\mathbb{F}_{p^{n}}[x]$. The monomials with possibly nonzero coefficients in both sides are $1, x, x^{p^{k}}$ and $x^{p^{n-k}}$. As the (polynomial) degrees 
of these univariate monomials are strictly smaller than $p^{n}$ and (40) holds for $p^{n}$ distinct elements $x \in \mathbb{F}_{p^{n}}$, the coefficients of the same degree univariate monomials in both sides of (40) are the same. Considering the coefficients of $x^{p^{n-k}}$ in both sides of (40) we conclude that

$$
\frac{\alpha}{L(\alpha)}=\frac{\alpha}{\tilde{L}(\alpha)} \text { for all } \alpha \in \mathbb{F}_{p^{n}}^{*}
$$

Hence $L(x)=\tilde{L}(x)$ for all $x \in \mathbb{F}_{p^{n}}$, namely

$$
a x^{p^{k}}+x^{p^{n-k}}+a_{0} x=b x^{p^{k}}+x^{p^{n-k}}+b_{0} x
$$

for all $x \in \mathbb{F}_{p^{n}}$. Now we consider the univariate monomials $x^{p^{k}}, x^{p^{n-k}}$ and $x \in \mathbb{F}_{p^{n}}[x]$ in both sides of (41). Again as the (polynomial) degrees of these univariate monomials are strictly smaller than $p^{n}$ and (41) holds for $p^{n}$ distinct elements $x \in \mathbb{F}_{p^{n}}$, the coefficients of $x^{p^{k}}$ in both sides of (41) are the same. Hence $a=b$. Considering the coefficients of $x$ in both sides of (41) we get $a_{0}=b_{0}$. This completes the proof of the statement that, for each $s$, the number of (distinct) functions in this class is at least the number in (33).

Using Theorems 10 and 11 we also get the following new GAPN functions of degree $p$

Example 4. Let $p$ be an odd prime. Let $n \geq 3$ and $1 \leq k \leq n-1$ be integers such that $\operatorname{gcd}(k, n)=1$. Let $H$ be the multiplicative subgroup of $\mathbb{F}_{p^{n}}^{*}$ with $|H|=\left(p^{n}-1\right) /(p-1)$.

- If $n$ is odd, then for $p>3$ let $a \in \mathbb{F}_{p^{n}}^{*} \backslash(H \cup-H)$.

- If $n$ is even, then let $a \in \mathbb{F}_{p^{n}}^{*} \backslash H$.

For $2 \leq s \leq p-1$ let $f: \mathbb{F}_{p^{n}} \rightarrow \mathbb{F}_{p^{n}}$ be the map given by

$$
f(x)=x^{p-s}\left(a x^{p^{k}}+x^{p^{n-k}}\right)^{s} .
$$

Then $f$ is a GAPN function of degree $p$ having exactly $s+1$ distinct terms. The number of functions in this class is exactly $(p-3) \frac{p^{n}-1}{p-1}$ if $n$ is odd, and $(p-2) \frac{p^{n}-1}{p-1}$ if $n$ is even. Indeed assume first that $n$ is odd. We have $(-1)^{\left(p^{n}-1\right) /(p-1)}=$ $(-1)^{1+p+\cdots+p^{n-1}}=(-1)^{n}=-1 \neq 1$ so that $-1 \notin H$. Therefore $H \cap-H=\emptyset$, $|H \cup-H|=2 \frac{p^{n}-1}{p-1}$ and the cardinality of $\mathbb{F}_{p^{n}}^{*} \backslash(H \cup-H)$ is

$$
p^{n}-1-2 \frac{p^{n}-1}{p-1}=(p-3) \frac{p^{n}-1}{p-1} .
$$

This implies that the number of distinct polynomials

$$
L(x)=a x^{p^{k}}+x^{p^{n-k}}
$$

with $a$ chosen as above is exactly $(p-3) \frac{p^{n}-1}{p-1}$. Let $L(x)=a x^{p^{k}}+x^{p^{n-k}}$ and $\tilde{L}(x)=b x^{p^{k}}+x^{p^{n-k}}$ be any two choices of such polynomials. Let $f(x)=$ 
$x^{p-s} L(x)^{s}$ and $\tilde{f}(x)=x^{p-s} \tilde{L}(x)^{s}$. As in Example 3 we obtain that, for each $s$, if $f=\tilde{f}$ as maps on $\mathbb{F}_{p^{n}}$, then $a=b$. This completes the proof of the statement that the number of functions in this class is exactly $(p-3) \frac{p^{n}-1}{p-1}$ if $n$ is odd. Next we assume $n$ is even. Then the cardinality of $\mathbb{F}_{p^{n}}^{*} \backslash H$ is

$$
p^{n}-1-\frac{p^{n}-1}{p-1}=(p-2) \frac{p^{n}-1}{p-1} .
$$

Similarly we prove that the number of functions in this class is exactly $(p-2) \frac{p^{n}-1}{p-1}$ if $n$ is even.

Theorem 11 item ii) implies a full classification of the functions of the form (42) if $n$ is odd.

Corollary 3. Let $p$ be an odd prime and assume $n$ is odd. Let $n \geq 3$ and $1 \leq k \leq n-1$ be integers such that $\operatorname{gcd}(k, n)=1$. For $a \in \mathbb{F}_{p^{n}}$ and $2 \leq s \leq p-1$, the function $f: \mathbb{F}_{p^{n}} \rightarrow \mathbb{F}_{p^{n}}$ given by

$$
f(x)=x^{p-s}\left(a x^{p^{k}}+x^{p^{n-k}}\right)^{s}
$$

is a GAPN function iff $a^{\left(p^{n}-1\right) /(p-1)} \notin\{1,-1\}$. In particular there exists a GAPN function $f$ of this form iff $p>3$.

Proof. One direction of the iff condition is already presented in Example 4. Conversely we prove the other direction using Theorem 11, in particular item ii).

The following class of GAPN functions follows from Theorem 10 rather directly.

Example 5. Let $p$ be an odd prime. Let $n \geq 3$ and $1 \leq k \leq n-1$ be integers such that $\operatorname{gcd}(k, n)=1$. For $a \in \mathbb{F}_{p^{n}}^{*}$ and $2 \leq s \leq p-1$ let $f: \mathbb{F}_{p^{n}} \rightarrow \mathbb{F}_{p^{n}}$ be the map given by

$$
f(x)=x^{p-s}\left(a x^{p^{k}}+x\right)^{s} .
$$

Then $f$ is a GAPN function of degree $p$ having exactly $s+1$ distinct terms iff $(-a)^{\left(p^{n}-1\right) /(p-1)} \neq 1$. The number of functions in this class is $(p-2) \frac{p^{n}-1}{p-1}$. Indeed the map on $\mathbb{F}_{p^{n}}^{*}$ given by $x \mapsto x^{p^{k}-1}$ is $(p-1)$-to- 1 as $\operatorname{gcd}\left(p^{k}-1, p^{n}-1\right)=p-1$. Hence the map $\psi: \mathbb{F}_{p^{n}}^{*} \rightarrow \mathbb{F}_{p^{n}}$ given by $\psi(x)=\left(a x^{p^{k}}+x\right) / x=a x^{p^{k}-1}+1$ is $(p-1)$-to- 1 as well. Using Theorem 10 it remains to prove that $L(x)=a x^{p^{k}}+x$ is permutation on $\mathbb{F}_{p^{n}}$. Assume $L$ is not a permutation. Then there exists $x \in \mathbb{F}_{p^{n}}^{*}$ such that $a x^{p^{k}-1}+1=0$, or equivalently $\frac{-1}{a} \in\left\{x^{p^{k}-1}: x \in \mathbb{F}_{p^{n}}^{*}\right\}$. Note that $\left\{x^{p^{k}-1}: x \in \mathbb{F}_{p^{n}}^{*}\right\}$ is the multiplicative group $H$ of $\mathbb{F}_{p^{n}}^{*}$ with $|H|=\frac{p^{n}-1}{p-1}$ and $\frac{-1}{a} \in H \Longleftrightarrow(-a)^{\left(p^{n}-1\right) /(p-1)}=1$. This completes the proof of the statement that $f$ is GAPN iff $(-a)^{\left(p^{n}-1\right) /(p-1)} \neq 1$. Moreover the cardinality of $\left\{a \in \mathbb{F}_{p^{n}}^{*}:(-a)^{\left(p^{n}-1\right) /(p-1)} \neq 1\right\}$ is

$$
p^{n}-1-\frac{p^{n}-1}{p-1}=(p-2) \frac{p^{n}-1}{p-1} .
$$


As in Examples 3 and 4 , we obtain that, for each $s$, there are exactly $(p-2) \frac{p^{n}-1}{p-1}$ functions in this class.

Finally we give a class using Theorems 9 and 11 .

Example 6. Let $p$ be an odd prime. Let $n \geq 3$ and $1 \leq k \leq n-1$ be integers such that $\operatorname{gcd}(k, n)=1$. Let $H$ be the multiplicative subgroup of $\mathbb{F}_{p^{n}}^{*}$ with $|H|=\left(p^{n}-1\right) /(p-1)$. For $a \in \mathbb{F}_{p^{n}}^{*}$ let $f_{1}: \mathbb{F}_{p^{n}} \rightarrow \mathbb{F}_{p^{n}}$ be the map given by

$$
f_{1}(x)=x^{p-1}\left(a x^{p^{k}}+x^{p^{n-k}}\right)
$$

and let $L_{1}(x)=a x^{p^{k}}+x^{p^{n-k}}$. Then we have the following:

i) If $n$ is odd, then

- $f_{1}$ is a GAPN function and $\operatorname{dim}_{\mathbb{F}_{p}} \operatorname{Ker} L_{1}=0$ iff $a \notin(H \cup-H)$,

- $f_{1}$ is a GAPN function and $\operatorname{dim}_{\mathbb{F}_{p}} \operatorname{Ker} L_{1}=1$ iff $a \in-H$.

ii) If $n$ is even and $a \notin H$, then $f_{1}$ is a GAPN function and $\operatorname{dim}_{\mathbb{F}_{p}} \operatorname{Ker} L_{1}=0$.

\section{Acknowledgement}

The authors thank to Royal Society for their support through the Newton Mobility Grant NI170158. During their visits, the authors benefitted from the hospitality of the Department of Computer Science, Loughborough University as well as the Department of Mathematics, Middle East Technical University, Ankara. We also thank the reviewers for careful comments which led to improvements to the paper.

\section{References}

1. T. P. Berger, A. Canteaut, P. Charpin, and Y. Laigle-Chapuy. On almost perfect nonlinear functions over $\mathbb{F}_{2}^{n}$. IEEE Transactions on Information Theory, 52:4160 4170, 2006.

2. E. Biham and A. Shamir. Differential cryptanalysis of DES-like cryptosystems. Journal of Cryptology, 4(1):3 - 72, 1991.

3. C. Blondeau and K. Nyberg. Perfect nonlinear functions and cryptography. Finite Fields and Their Applications, 32:120 - 147, 2015.

4. I. Dinur and A. Shamir. Cube attacks on tweakable black box polynomials. In EUROCRYPT, pages 278-299, 2009.

5. X-D. Hou. Solution to a problem of S. Payne. Proceedings of the American Mathematical Society, 132:1 - 6, 2003.

6. X. Hou. Two classes of permutation polynomials over finite fields. Journal of Combinatorial Theory, Series A, 118(2):448 - 454, 2011.

7. X. Hou. A new approach to permutation polynomials over finite fields. Finite Fields and Their Applications, 18(3):492 - 521, 2012. 
8. M. Kuroda. Monomial generalized almost perfect nonlinear functions. arXiv:1708.00686, 2017

9. M. Kuroda and J. Tsujie. A generalization of APN functions for odd characteristic. Finite Fields and Their Applications, 47:64 - 84, 2017.

10. X. Lai. Higher order derivatives and differential cryptanalysis. In R. E. Blahut, D. J. Costello, Jr., U. Maurer, and T Mittelholzer, editors, Communications and Cryptography, volume 276 of The Springer International Series in Engineering and Computer Science, pages 227-233. Springer, 1994.

11. R. Lidl, H. Niederreiter. Finite Fields, Second Edition, Encyclopedia of Mathematics and its Applications, 20, Cambridge University Press, Cambridge, 1997.

12. K. Nyberg. Differentially uniform mappings for cryptography. in:T. Helleseth (Ed.), Advances in Cryptology EUROCRYPT93: Workshop on the Theory and Application of Cryptographic Techniques, (Lofthus, Norway, May 23-27, 1993), Lecture Notes in Comput. Sci., Springer, Berlin, 765:55-64, 1994.

13. A. Pott. Almost perfect and planar functions. Designs, Codes and Cryptography, $78: 141-195,2016$.

14. A. Sălăgean, R. Winter, M. Mandache-Sălăgean and R.C.W. Phan, Higher order differentiation over finite fields with applications to generalising the cube attack. Designs, Codes and Cryptography, 84:425-449, 2017.

15. M. Vielhaber. Breaking ONE.FIVIUM by AIDA an algebraic IV differential attack. Cryptology ePrint Archive, Report 2007/413, 2007. urlhttp://eprint.iacr.org/.

16. Z. Zha, L. Hu, and Z. Zhang. Three new classes of generalized almost perfect nonlinear power functions. Finite Fields and Their Applications, 53:254 - 266, 2018. 